\title{
Annotationes Epigraphicae IX \\ Zu einigen Inschriften aus den römischen Provinzen Germania inferior und Germania superior*
}

\author{
Andreas Kakoschke
}

I.

Nördlich von Euskirchen-Rheder (Kr. Euskirchen) im Erfttal entdeckte man im Jahre 1841 in einem römerzeitlichen Grab einen kleinen Altar (Höhe $30 \mathrm{~cm}-$ Breite $18 \mathrm{~cm}$ - Tiefe $9 \mathrm{~cm}$ ) aus Kalkstein (Abb.1). ${ }^{1}$ Die stark abgeriebene Inschrift des Steins, die der Zeit zwischen 170 und 230 n. Chr. zugeordnet werden kann, fand Aufnahme in das CIL und wird einige Jahre später von LEHNER entsprechend den Angaben im CIL wie folgt gelesen: ${ }^{2}$

\author{
D(eo) I(nvicto) M(ithrae) Fir- \\ mino vo- \\ tum refe- \\ ret Ius- \\ $5 \quad$ tini Pat- \\ erna \\ $v$ (otum) [s(olvit) l(ibens) m(erito)?]
}

Bereits HETTNER hatte (aufgrund eines mündlichen Hinweises durch BÜCHELER) darauf verwiesen, dass mit den Worten Firmino bis Paterna ein Hexameter vorliegt. HETTNERs Auffassung zufolge, die von LEHNER geteilt wird, erklären sich die Formen Firmino (statt pro salute Firmini) und referet (statt refert) sowie ,die Vorstellung des Genitivs" im Namen Iustini Paterna durch das beabsichtigte Versmaß. ${ }^{3}$

Nach CLAUSs bleibt die Bedeutung von Iustini Paterna „problematisch“, da es sich möglicherweise um eine ,,aufgrund des Versmaßes stark verunstaltete Namensform“ handeln kann. Entgegen LEHNER, der Iustini Paterna mit „Paterna, Tochter des Justinus“ wiedergibt, übersetzt Clauss den Namen mit „Paterna, Gattin des Iustinus". ${ }^{4}$ Dabei folgt Clauss den Ausführungen WIEGELS', der in Paterna ebenfalls die Gattin eines Iustinus erblickt und die Frau zudem mit einer in Rheder bezeugten

\footnotetext{
* Für die Bereitstellung einiger Photos danke ich Herrn Prof. Dr. R. Wiegels und Herrn Prof. Dr. K. Matijević (CIL XIII/2-Projekt, Universitäten Trier/Flensburg) sowie Herrn Dr. O. Harl (Universität Wien) und Herrn M. Perse (Museum Jülich). Bei den Herausgebern der FeRA, bei Herrn Prof. Dr. K. Matijević und Herrn Dr. P. Probst (Hamburg), bedanke ich mich wiederum für einige wichtige Hinweise. - Ligaturen bzw. Nexus werden im Folgenden durch unterstrichene Buchstaben wiedergegeben. - Die bisherigen Annotationes Epigraphicae erschienen in den vorhergehenden Ausgaben der FeRA. Die Annotationes Epigraphicae III finden sich im GFA 19 (2015), 179-197.

${ }^{1} \mathrm{Zu}$ den Fundumständen s. J. KATZFEY, BJb 1 (1842), 128.

${ }^{2}$ LEHNER 1918, 104. Nr.220 = CIL XIII 7958/9. - Gleich lautende Lesungen bieten VERMASEREN 1960, 53-54. Nr.1034, WeISGERBER 1968, 28. Nr.183, EDCS Nr.11100183. - Der Stein befindet sich heute im Rheinischen Landesmuseum in Bonn (Inv.-Nr.U 71).

${ }^{3}$ HETTNER 1876, 25-26. Nr.71.

${ }^{4}$ CLAUSS 1992, 99.
} 
Iulia Paterna ${ }^{5}$ identifiziert. ${ }^{6}$ Eine entsprechende Vermutung findet sich bereits bei LEHNER $^{7}$ und später bei SCHWERTHEIM. ${ }^{8}$

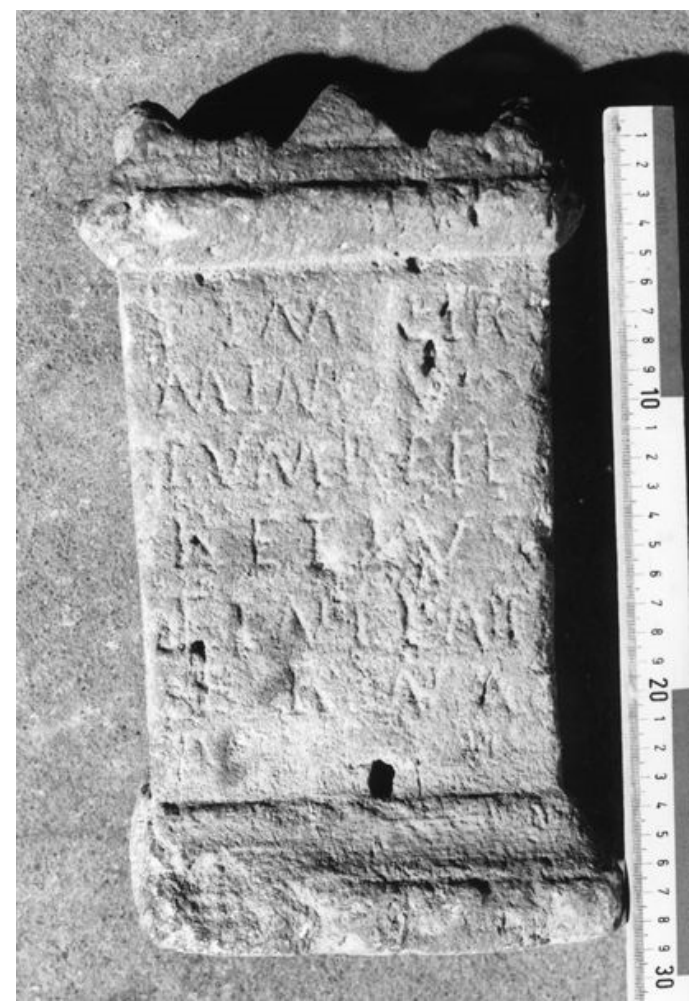

Abb.1: Weihinschrift aus Euskirchen-Rheder: CIL XIII 7958/59

(C) CIL XIII/2-Projekt, Universitäten Trier/Flensburg)

Ein Blick auf weitere Inschriften aus den germanischen Provinzen zeigt, dass die Form Iustini Paterna keineswegs außergewöhnlich und entgegen den bisher referierten Auffassungen offenbar mit Iustini(a) Paterna wiederzugeben ist. So findet sich im benachbarten Bonn-Endenich die Weihinschrift eines Attici Maternus. ${ }^{9}$ Da der Mann ein miles legionis Minerviae war, also mit Sicherheit ein (Pseudo-)Gentiliz führte, lautete sein Name höchstwahrscheinlich Atticius Maternus. Entsprechendes gilt für einen beneficiarius consularis aus Stuttgart-Bad Cannstatt, der seinen Namen auf einer Weihinschrift mit Sereni Atticus angibt. ${ }^{10}$ Daher sollte auch der Name Iustini Paterna als Iustini(a) Paterna gelesen werden. ${ }^{11}$ Diese Auffassung ist keinesfalls neu. Eine entsprechende Lesung bot bereits der Ersteditor der Inschrift, LERSCH, ${ }^{12}$ und später RIESE. ${ }^{13}$ Die Lesungen wurden jedoch nicht wieder aufgegriffen.

Clauss betont berechtigterweise, dass mit der Weihung aus Rheder die bislang einzige Weihung einer Frau im Mithras-Kult vorliegt. ${ }^{14}$ Der Umstand spricht

\footnotetext{
${ }^{5}$ CIL XIII $7960=$ LEHNER 1918, 315. Nr.784.

${ }^{6}$ R. WIEGELS, Freiburger Universitätsblätter 65 (1979), 61. Anm.80. Vgl. auch ENGSTER 2002, 444. Anm.6.

${ }^{7}$ LEHNER 1918, 104. Nr.220.

${ }^{8}$ SCHWERTHEIM 1974, 39-40. Nr.41.

${ }^{9}$ CIL XIII 8003a = LEHNER 1918, 192. Nr.509.

10 1. N. 205. Nr.345 = AE 1927, 66.

${ }^{11}$ Zur Problematik dieser Namensform s. A. KAKOSCHKE, FeRA 26 (2015), 14-20 (mit weiteren Beispielfällen).

${ }^{12}$ L. LERSCH, BJb 1 (1842), 85.

${ }^{13}$ RIESE 1914, 348. Nr.3410.

${ }^{14}$ Clauss 1992, 99. Vgl. auch Engster 2002, 444. Anm.6.
} 
jedoch nicht gegen die vorgeschlagene Lesung des Dedikantennamens, da, wie WIEGELS zu Recht feststellt, die Frau zumindest nicht zwingend zur Kultgemeinde gehört haben muss. ${ }^{15}$ Auf jeden Fall lässt sich die jüngst von GRIFFITH vorgeschlagene Lesung Iustini(us) Patern(i)a(nus) oder Iustini(us) Patern $\{a\}(u s)$ $v$ [et(eranus)], die eine starke Korrektur des Textes erfordert, wohl ausschließen. ${ }^{16}$ Sicherlich gehört auch das V der letzten Zeile am ehesten zur Schlussformel VSLM. GRIFFITH weist berechtigterweise darauf hin, dass damit das votum zweimal genannt wird. ${ }^{17}$ Allerdings ist auch dieser Umstand nicht außergewöhnlich, da z.B. eine Weihinschrift aus Mudau-Steinbach ebenfalls zweimal das votum nennt ${ }^{18}$ und eine Weihinschrift aus Colijnsplaat/Ganuenta die abgekürzte Formel VSLM (offenbar aufgrund einer zweiten Beschriftung des Steins) am Ende gleich zweimal aufweist. ${ }^{19} \mathrm{Zu}$ verwerfen ist sicherlich auch die Auflösung der ersten Buchstaben zu D(is) I(nferis) $M$ (anibus), wie es GRIFFITH angesichts der Fundumstände am Rande erwägt. ${ }^{20}$ Bei der Inschrift handelt es sich schließlich angesichts der Wendung votum referet ohne Zweifel um eine Weihinschrift, die erst in zweiter Verwendung zum Bau einer Grabeinfassung diente. ${ }^{21}$

Somit wurde die Inschrift für Mithras aus Euskirchen-Rheder offensichtlich von einer Frau namens Iustini(a) Paterna für eine Person namens Firminus, (pro) Firmino, dediziert. ${ }^{22}$

\section{II.}

Eine ungewöhnliche Wortfolge weist auch die folgende Inschrift auf. Die trotz einer größeren Verletzung gut lesbare Weihinschrift (Höhe $105 \mathrm{~cm}$ - Breite $33 \mathrm{~cm}$ - Tiefe $19 \mathrm{~cm}$ ) eines Altars aus Sandstein (Abb.2) fand sich im Jahre 1866 in Stettfeld, einem Ortsteil der Gemeinde Ubstadt-Weiher (Ldkr. Karlsruhe). Im CIL wird der Text, der in die Zeit um $200 \mathrm{n}$. Chr. datiert werden kann, von ZANGEMEISTER mit leichten Zweifeln (,fortasse“) wie folgt wiedergegeben: ${ }^{23}$

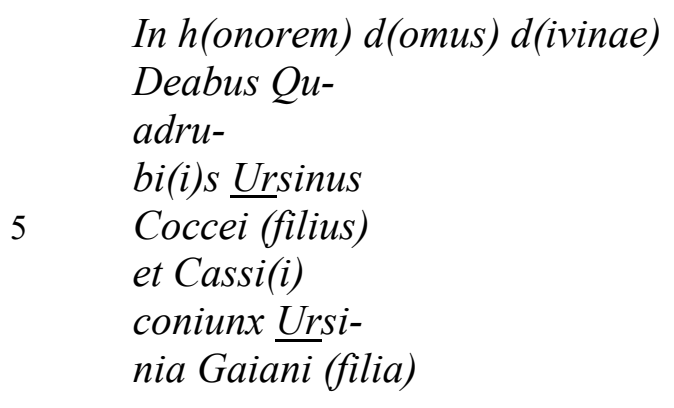

\footnotetext{
${ }^{15}$ R. WIEGELS, Freiburger Universitätsblätter 65 (1979), 61. Anm.80.

${ }^{16}$ A. B. GRIFFITH, Numen 53/1 (2006), 58.

${ }^{17}$ A. B. GRIFFITH, Numen 53/1 (2006), 58.

${ }^{18}$ CIL XIII 6520 = EDCS Nr.11000546: Dian(a)e / votum / Vitalis / pro se / et su\{u\}os / v(otum) s(olvit) l(ibens) l(aetus) m(erito).

${ }^{19}$ AE 2001, 1505 = EDH Nr.HD047386: --- / C(aius) Vitalinius / Genialis / v(otum) s(olvit) l(ibens) m(erito) / v(otum) s(olvit) l(ibens) m(erito). - Aus dem Bestand der außerhalb der germanischen Provinzen bezeugten Inschriften ist vor allem auf eine Mithrasinschrift aus Zgornja Pohanca im Grenzgebiet zwischen Noricum und Pannonien zu verweisen (LUPA Nr.6137 = EDH Nr.HD066967): D(eo) I(nvicto) M(ithrae) / Metilius / Iustianus / vot(um) sol(vit) pr/o salute sua / v(otum) s(olvit) l(ibens) l(aetus) m(erito).

${ }^{20}$ S. auch schon CLE II/3. 36. Nr.1957.

${ }^{21}$ Vgl. J. KATZFEY, BJb 1 (1842), 128, SCHWERTHEIM 1974, 39. Nr.41.

${ }^{22}$ Von SPICKERMANN 1994 wurde die Inschrift jedoch nicht berücksichtigt.

${ }^{23}$ CIL XIII 6343. - Zur Inschrift s. auch BRAMBACH 1867, XXXI. Nr.2061, RIESE 1914, 354. Nr.3487, EDCS Nr.11000353 (mit Photos), EDH Nr.HD036518 (mit Photos), LUPA Nr.21613 (mit Photos). Die Inschrift befindet sich heute im Reiss-Engelhorn-Museum in Mannheim (Inv.-Nr.Baumann 22).
} 


\section{ex voto \\ 10 \\ posuerunt}

Gemäß ZANGEMEISTER wurde die Inschrift also von Ursinus, dem Sohn des Cocceius, und von Ursinia, der Tocher des Gaianus, gesetzt. Wobei Ursinia die Gattin des Cassius war. ${ }^{24}$ Im Kommentar zur Inschrift bietet das CIL ferner einen Hinweis MOMMSENs, demzufolge der Steinmetz in Zeile 6 möglicherweise fehlerhafterweise ET CASSI statt CASSI ET eingeschlagen hat. Die Anregung wird später auch von HAUG aufgegriffen. Laut HAUG wurde die Inschrift demnach von „Ursinus, Sohn des Coccejus Cassus(!)“ und von seiner Gattin, „Ursinia, Tochter des Gajanus“" gestiftet. ${ }^{25}$

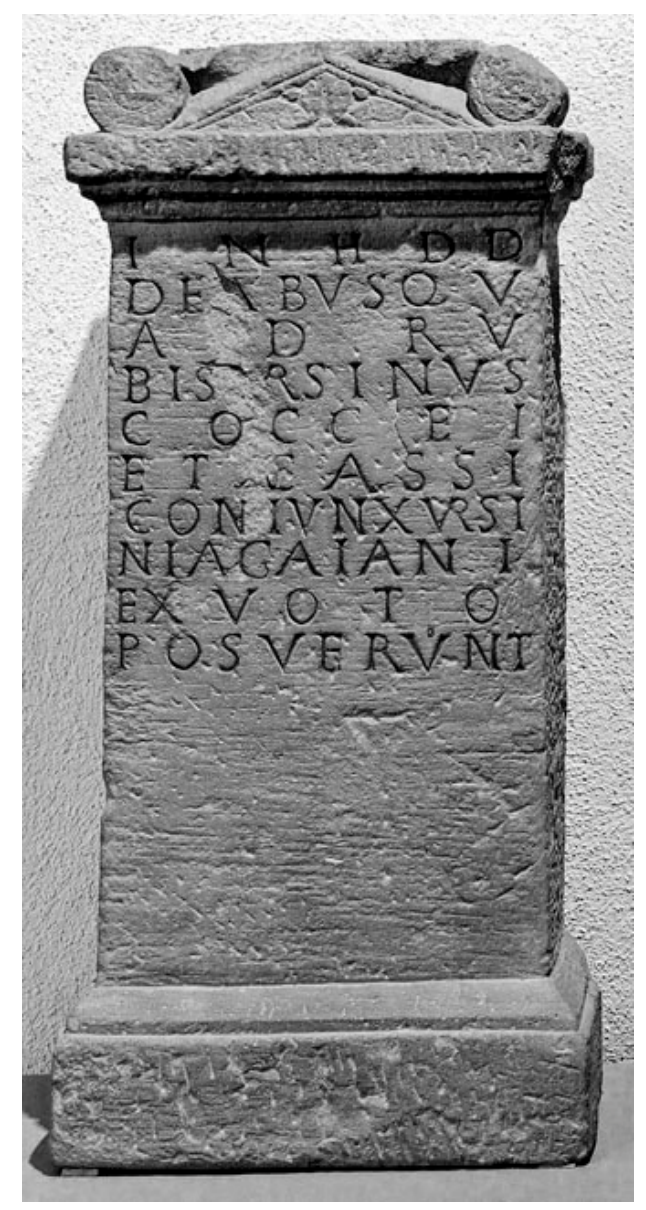

Abb.2: Weihinschrift aus Stettfeld: CIL XIII 6343

[C Ortolf Harl, Wien (LUPA)]

Der Interpretation HAUGs und MommSENs neigt auch WIEGELS zu, da der Altar in diesem Fall - wie häufig bezeugt - von einem Ehepaar dediziert wurde. Dagegen bleibt der Lesung ZANGEMEISTERs zufolge das Verhältnis der Personen zueinander unklar. Als etwas störend empfindet WIEGELS jedoch neben dem Eingriff in den Text das allein stehende Cognomen des Dedikanten, Ursinus, während der Name des Vaters offensichtlich mit Gentiliz und Cognomen, Cocceius Cassius, wiedergegeben wird, sowie die ungewöhnliche Voranstellung von coniunx. Zu den Namen der vermeintlichen Eheleute äußert sich WIEGELS wie folgt: „Ursinus ist zwar allenthalben

\footnotetext{
${ }^{24}$ Vgl. auch M. IHM, BJb 83 (1887), 132. Nr.180, BAUMANN 1890, 16. Nr.22, GROPENGIEßER 1975, 13. Nr.13. Abb.13 (Photo), M. MATTERn, AKB 28 (1998), 612. Nr.25.

${ }^{25}$ F. HAUG, in: WAGNER 1911, 178. Die alternative Lesung wird auch von GROPENGIERER 1975, 13. Nr.13. Abb.13 (Photo) angemerkt.
} 
nicht selten, auffallender jedoch der Name der Frau, Ursinia, also entsprechend demjenigen des (Ehe-?)Mannes, jedoch mit i-Erweiterung in der Weise eines Gentiles, vielleicht als Pseudogentile bei Ehelichung von der von einem peregrinen Vater Abstammenden angenommen." 26

Bei den Dedikanten der Inschrift, den im Nominativ genannten Personen, Ursinus und Ursinia, wird es sich m.E. aufgrund des Pseudogentilnomens Ursinia höchstwahrscheinlich nicht um Eheleute, sondern um Vater und Tochter handeln. Der Umstand war für den antiken Leser, der mit patronymischen Bildungen vertraut war, sicher unübersehbar. Daher konnte auf eine ausdrückliche Angabe im Text verzichtet werden.

Die in den germanischen Provinzen bezeugten Ehefrauen tragen in der Regel einen eigenen Namen, der sich vom Namen ihres Mannes unterscheidet. Dabei führen die Frauen entweder einen Namen der Form „Gentiliz + Cognomen“ oder „Individualnamen mit peregriner Filiation“. Bei Ehepaaren mit identischen Gentilnomina kann die Frau das Gentiliz des Mannes angenommen haben oder es handelt sich bei dem Ehepaar - obwohl ein eindeutiger Hinweis im Text fehlt - um Freigelassene desselben Patrons. ${ }^{27}$ Darüber hinaus sind aus den germanischen Provinzen einige Belege für Ehefrauen mit nur einem Namen bekannt. In den meisten Fällen wird der Name der Frau dabei wohl nur mit einem Nomen simplex oder einem Cognomen angegeben. ${ }^{28}$ Entweder war die Frau somit eine Peregrine, sie trug also nur einen einfachen Namen, oder das (Pseudo-)Gentilnomen der römischen Bürgerin wurde einfach nicht genannt. ${ }^{29}$ In einigen Fällen hat man das (Pseudo-)Gentiliz der Frau vielleicht unter-

${ }^{26}$ Wiegels 2010, 243-244. Abb.5 (Photo) [= R. Wiegels, FBW 17/1 (1992), 384-386. Abb.5 (Photo)]. Vgl. auch SPICKERMANN 1994, 274. Nr.1.

${ }_{27}$ Vgl. SpickermanN 1994, 16, Wiegels 2010, 203. Anm.28. - Zwei Freigelassene eines Patrons waren z.B. der aus Gebenstorf bei Windisch/Vindonissa bekannte medicus legionis XXII Ti. Claudius Hymnus und seine Frau Claudia Quieta (CIL XIII 5208 = LAZZARO 1993, 122-123. Nr.84). Entsprechendes gilt wahrscheinlich auch für M. Porcius M. lib. Crescens und seine Frau Porcia Mansueta (CIL XIII 11635 = LAZZARO 1993, 164. Nr.153; Strasbourg/Argentorate). - Ehepaare mit identischen Namen bezeugt z.B. die Grabinschrift CIL XIII 6000 = LAZZARO 1993, 165-166. Nr.155 [Saverne/Tres Tabernae; Cornel(ia) Divixta und Cor(nelius) Iuvenilis]. S. ferner CIL XIII 5107 = LAZZARO 1993, 115. Nr.71 (Avenches/Aventicum; Otacilia Faventina und der VIvir M. Otacilius), 5132 [Avenches/ Aventicum; Flavia Severilla und Flav(ius) Severinus], 5135 (Avenches/Aventicum; D. Iulius Iunus und Iulia Titullina), 6311 (Baden-Baden/Aquae; Gnoria Amabilis und Gnorius Primigenius), $6368=$ LAZZARO 1993, 172. Nr.164 (Rottenburg/Sumelocenna; Iulia Severina und D. Iulius Severus), $7112=$ LAZZARO 1993, 202. Nr.215 [Mainz/Mogontiacum; Speratius Hermodurus und Speratia Iuliana], 7116 [Mainz/Mogontiacum; Titinia Amm[...] und Titin(ius) Castus], 7520 = LAZZARO 1993, 212. Nr.236 [Bingen-Bingerbrück; Iulia Quintia und Ti. Iul(ius) Eunus].

${ }^{28}$ Verwiesen sei u.a. auf folgende Inschriften: CIL XIII 3620 [Namur/Namurcum; Amma, Frau des Acceptus Victoris (f.)], 5153 [Amsoldingen; Asinula, Frau des Severius Commendatus], 6125 [Hoheburg; Prima, Frau des [...]nucco Cacussonis (f.)], 6201 (Schwarzerden; Cinna, Frau des Tertius Tertinus), 6310 (Baden-Baden; Domestica, Frau des Q. Valerius Pruso), 6393 [Neckargemünd-Kleingemünd; Meddila, Frau des Petus Atici (f.)], 6534 (Murrhardt; Victorina, Frau des Meddillius Carantus), 6970 [Mainz/Mogontiacum; Claudi\{n?\}a, Frau des Sammonius Paterclus], 7572 (Wiesbaden/Aquae Mattiacorum, Romula, Frau des Firmius Firminus), $8342=$ IKöln $^{2}$ 347-348. Nr.417 (Köln/CCAA; Demionca, Frau des Gatus Cabiri f.), $8409=$ IKöln² 428-429. Nr.546 (Köln/CCAA; Exomna, Frau des Ocellio Illanuonis f.), 1. N. 71. Nr.215 (Mainz/Mogontiacum; Haledona, Frau des L. Aetilius [---]), 2. N. 76. Nr.82 (Odernheim am Glan; Aprilla, Frau des T. Assonius Sappo), 94. Nr.144 (Kierdorf; Vimpa, Frau des A. Volsonius A. f. Cla. Paulus), 4. N. 488-489. Nr.58 (Stockstadt; Cisso, Frau des Attius Tertius), ILTG 436 (Langensoultzbach; Attiola, Frau des [Dom?]itius Silvinus), AE 1982, 722 (Kirchberg/Dumnissus, Connouga, Frau des Maximius Acceptus), 1996, 1143 (Augst/ Augusta Raurica; Eucerilla, Frau des Bellinius Rhenicus), 2012, 993 [Leuzigen; Florentina, Frau des Tib. Sanctius Ingens], LUPA Nr.27113 [Heidelberg-Wieblingen; Siora, Frau des Senecio Ianuari (f.)].

${ }^{29}$ Für den letzteren Fall kann nur auf wenige Belege aus den germanischen Provinzen verwiesen werden. In zwei Fällen handelt es sich um auswärtige Frauen von höhergestellten Persönlichkeiten: Modestiana, Frau eines legatus Augusti (CIL XIII 6806, Mainz/Mogontiacum) und Attica, Frau des

http://www.fera-journal.eu 
schlagen, da es mit dem Namen des Mannes identisch war. ${ }^{30}$ Darüber hinaus kann es sich bei einigen weiblichen Einzelnamen auch um ein allein stehendes Gentil- oder Pseudogentilnomen handeln. ${ }^{31}$ Allerdings war der Gebrauch eines einfachen Gentilnomens als Name für eine Frau in der Kaiserzeit generell bereits veraltet. ${ }^{32}$

Hinsichtlich des Frauennamens in der Inschrift aus Stettfeld lässt sich noch festhalten, dass die Form Ursinia/Ursinius nicht weiter als Cognomen bzw. Nomen simplex bezeugt ist. Aus den zwei germanischen Provinzen sind jedoch sehr wohl weitere Belege für das Pseudogentiliz Ursinius bekannt. ${ }^{33}$

Nach dem bisher Gesagten wird Ursinia wohl nicht die Tochter des Gaianus gewesen sein. Unter der Annahme einer recht ungewöhnlichen Wortstellung, die aber auch in weiteren Inschriften anzutreffen ist, ${ }^{34}$ wird man den Genitiv Gaiani am ehesten zu Cassi(i) ziehen. Demnach darf man in Ursinia wahrscheinlich die Frau eines Cassius Gaianus (oder eines Gaianius ${ }^{35}$ Cassius) erblicken. Nicht zu klären ist, ob die auffällige Inversion der Worte in der Inschrift absichtlich erfolgte oder ein Steinmetzfehler vorliegt. ${ }^{36}$

Es bleibt darauf zu verweisen, dass Ursinus Coccei(i) (filius) einen Namen der Form „Individualname mit peregriner Filiation“ führt. Cocceius ist zwar in erster Linie ein (weit verbreitetes) Gentiliz, doch finden sich im gesamten Imperium vereinzelte Belege für das Cognomen Cocceius. ${ }^{37}$

Konsulars A. Didius Gallus Fabricius Veiento (CIL XIII 7253, Klein-Winternheim). Ein Gentiliz (Publicia?) trug mit Sicherheit ferner die liberta publica Decorata, die Frau des servus Gratinus (2. N. 72. Nr.75, Altrip/Alta Ripa).

${ }^{30}$ Gelegentlicht wurden Frauen (als Dedikantin oder als Verstorbene) namentlich auch gar nicht genannt: CIL XIII 6190 (Oberstaufenbach; Visionio Iaso et coniugi), 6985 [Mainz/Mogontiacum; coniux eiius(!) et Potentinius Victor], 8357 = IKöln ${ }^{2} 374$. Nr.448 (Köln/CCAA; Adnamatius Speratus coniugi \{coniug(i)\} dulcissim(a)e adque incom[parab(ili)]), 8644 (Xanten/CUT; et coniugi), 8772 [Ruimel; et co[niugi] kariss(imae) et piis(imae)], 11703 (Heidenburg; Poppausio Cossetioni et coniugi eius).

${ }^{31}$ Verwiesen sei u.a. auf folgende Inschriften: CIL XIII 5284 = LAZZARO 1993, 127-128. Nr.91 (Augst/ Augusta Raurica; Castia, Frau des Castius Peregrinus), 5383 (Besançon/Vesontio; [Vi]rginia, Frau des Marius Vitalis), 6237 [Worms-Weinsheim/Borbetomagus; Albisia, Frau des T(itius?) Tummo], 7685 [Andernach/Antunnacum; Iulia, Frau des Q. Cellius Firmus], 4. N. 526. Nr.141 [Mayen; Restia, Frau des Amminius Adnamatus].

32 KAJANTO 1977, 152-153.

33 CIL XIII 6484 (Bad Wimpfen), 1. N. 92. Nr.276 (Bonn/Bonna), Stümpel, B. In: MZ 79/80 (1984/85), 262 (Essenheim). - Ein Pseudogentiliz darf man vielleicht auch im Namen der aus Sankt Julian bekannten Perpetuia vermuten (CIL XIII $6178+6179=11706=$ LUPA Nr.26587). Perpetuius ist ansonsten ebenfalls nur als Pseudogentiliz bezeugt. Das OPEL III 133 und KAKOSCHKE 2006, 306. GN 940/5 führen den Beleg aus Sankt Julian daher als (Pseudo-)Gentiliz.

${ }^{34}$ S. z.B. CIL XIII 6024 [Ingwiller; August(a)e fili(a)e Se/cconis ...], 6087 [Rheinzabern/Tabernae; Silvano / Tetto/serus / fi(lius) Tacit(i) ...], 6876 [Mainz/Mogontiacum; L(ucius) Vinicius L(uci) f(ilius) Gal(eria tribu) domo Modestus / Lugud(uno) ...], 6942 [Mainz/Mogontiacum; Miles leg(ionis) / XVI 7 (centuriae) Viato/ris Sex(tus) / Lartidius / Sex(ti) f(ilius) Vel(ina tribu) / Pistoris ...], 7029 [Mainz/Mogontiacum; C(aius) Romanius / eq(ues) alae Norico(rum) / Claud(ia tribu) Capito / Celeia ...], 7579 [Wiesbaden/Aquae Mattiacorum; Muranus / eq(ues) ala(e) I Flavia(e) / Androuri f(ilius) ...], 3. N. 196-197. Nr.201 [Bonn/Bonna; Nemesi Dia/nae Publius / Publi (filius) Claudia / Savaria Acu/leiensis Opponi(us) / Iustus ...].

${ }^{35}$ Ein vom Cognomen Gaius oder Gaianus abgeleitetes Pseudogentiliz Gaianius ist entgegen WIEGELS 2010, 244. Anm.28 bis heute nicht bezeugt, aber durchaus denkbar.

${ }^{36}$ Sollte der Ehemann der Ursinia den Namen Gaiani(us) Cassi(us) getragen haben, bietet sich eine mögliche Erklärung für den vorliegenden Text an. Vielleicht sah die Vorlage für den Steinmetzen etwa wie folgt aus: INHDD / DEABVS / QVADRVBIS / VRSINVS COCCEI ET $/ 5$ VRSINIA GAIANI / CASSI CONIVNX / EX VOTO / POSVERVNT. Beim Einschlagen hielt sich der Steinmetz nicht an die vorgegebene Zeilenaufteilung, vertauschte versehentlich die Zeilen 5 und 6 der Vorlage und gelangte zu dem bekannten Ergebnis. Zu vertauschten Zeilen in Inschriften s. auch die Hinweise bei KAKOSCHKe 2014, 12. Anm.5, A. KAKOSCHKE, FeRA 27 (2015), 20-24.

${ }^{37}$ Zum Namen s. KAKOSCHKE 2007, 252. CN 862, OPEL II 67, DELAMARRE 2007, 69.

http://www.fera-journal.eu 
III.

Im Jahre 1842 entdeckte man in Augst/Augusta Raurica (Kanton Basel-Landschaft), genauer im Gräberfeld Rheinstraße (Region 10,B), eine fragmentarische Grabplatte (Höhe 46,5 - Breite 33,5 cm - Tiefe $14 \mathrm{~cm}$ ) aus Sandstein (Abb.3). Die Inschrift, die wohl dem 3. Jahrhundert n. Chr. angehört, ist von niederer Qualität. WALSER gibt den Text wie folgt wieder. ${ }^{38}$

\section{Marini(o) \\ Cossi \\ filio Attili- \\ [anus? ---]}

Demnach liest WALSER einen Namen mit cognominaler Filiation: „Marinius Attilianus, Sohn des Cossus" ${ }^{39}$ Ebenso wie WALSER lesen den Text P.-A. SCHWARZ und A. R. Furger, übersetzen jedoch „Für Marinius, den Sohn des Cossus, hat Attilianus ...". 40

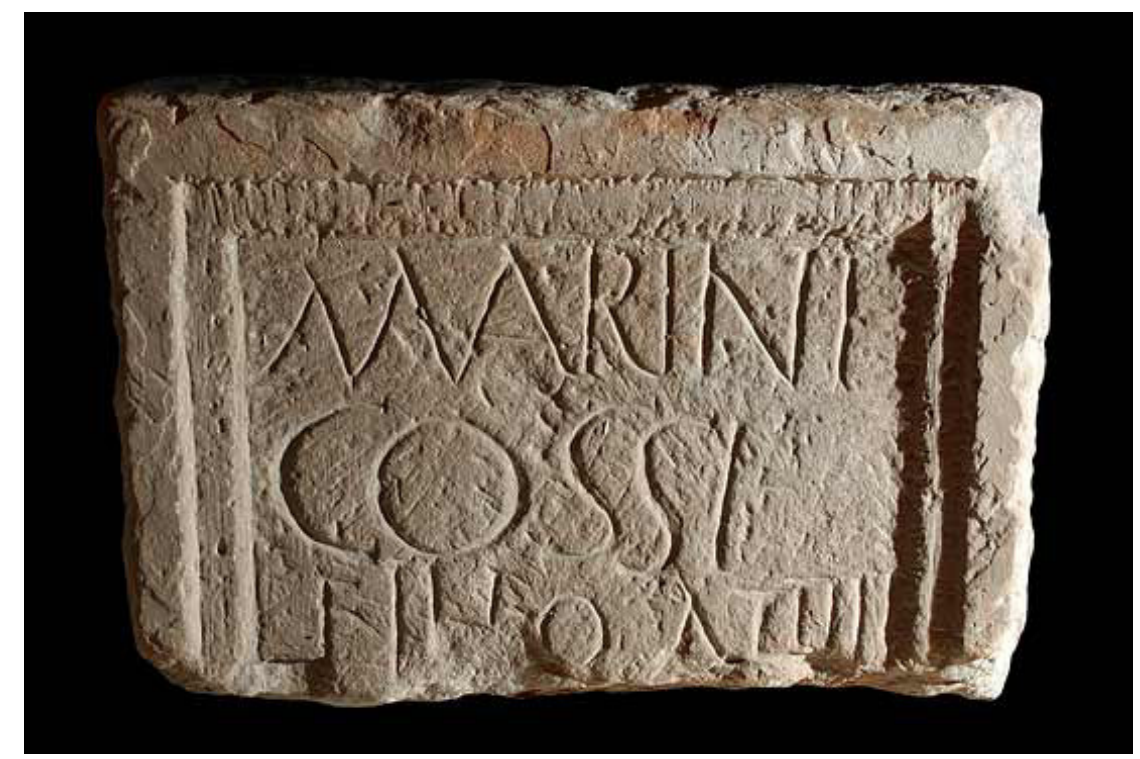

Abb.3: Fragmentarische Grabinschrift aus Augst/Augusta Raurica: CIL XIII 5291 [C Ortolf Harl, Wien (LUPA)]

Diese Lesungen können m.E. allerdings nur schwerlich zutreffen, da die Deutung des Namens MARINI als Dativ Marini(o) sehr unwahrscheinlich ist. Im gesamten Namenmaterial der germanischen Provinzen findet sich kein vergleichbarer Fall für

\footnotetext{
${ }^{38}$ WALSER 1980, 228-229. Nr.222 (mit Photo). Hier mit einer leicht modifizierten Wiedergabe. - Der Stein befindet sich heute im Römermuseum in Augst (Inv.-Nr.1904.132).

39 S. auch KAKOSCHKE 2006, 259-260. GN 745/2, B. HARTMANN, JberAugst 34 (2013), 132, 134, LUPA Nr.8433.

${ }^{40}$ Schwarz/Furger 1988, 10-11. Abb.4 (Photo). Vgl. auch ICH 60. Nr.294. Laut Index (ICH p.122) favorisiert MOMMSEN die Lesung „Marinus (Cossi f.)“. Entsprechend führt STÄHELIN 1948, 496. Anm.7 den Namen „Marinus, Sohn eines Cossus“. - Lediglich eine Textwiedergabe bieten: RIESE 1914, 382. Nr.3841, EDCS Nr.10800695.
} 
diese Lesung. ${ }^{41}$ So liegt im vorliegenden Fall die Lesung eines in dieser Form vielfach bezeugten Genitivs viel näher: Marini(i) Cossi. ${ }^{42}$ Zwar ist die Lesung einer unvollständig erhaltenen Inschrift immer mit Unwägbarkeiten verbunden, doch galt die Grabinschrift aus Augst/Augusta Raurica wohl Attili[anus?], dem Sohn des Marinius Cossus. Dabei ist die Wortstellung auf den ersten Blick ungewöhnlich. Man hätte eher die Formulierung Attiliano Marini(i) Cossi filio oder ähnlich erwartet. Wie die zuvor besprochene Inschrift aus Stettfeld jedoch zeigt, kann eine Inversion nicht ausgeschlossen werden. Zudem demonstrieren Namen wie T. Flavi(i) Fortunati lib(ertus) Primitius einer Weihinschrift aus Domburg ${ }^{43}$ und L(ucii) et C(aii) et Sex(ti) Valeriorum l(ibertus) Servandus einer Grabinschrift aus Mainz/Mogontiacum, ${ }^{44}$ dass ein vorangestellter Gentiv grundsätzlich durchaus möglich ist. Dafür sprechen auch die schon genannten Namen Iustini(a) Paterna, ${ }^{45}$ Attici(us) Maternus ${ }^{46}$ und Sereni(us) Atticus,${ }^{47}$ die auch die Lesungen Iustini (filia) Paterna, Attici (filius) Maternus und Sereni (filius) Atticus nicht ganz ausschließen. ${ }^{48}$

IV.

Im Jahre 1785 fand sich in Titz-Rödingen (Kreis Düren) ein in mehrere Teile zerbrochener fragmentarischer Weihestein (Höhe $102 \mathrm{~cm}$ - Breite $66 \mathrm{~cm}$ - Tiefe ca. 30 $\mathrm{cm})$ aus gelblichgrauem Sandstein mit einer Matronendarstellung über dem Inschriftfeld. Der Stein gelangte unmittelbar nach seiner Auffindung mit anderen Inschriftensteinen ${ }^{49}$ ins Kurfürstliche Antiquarium nach Mannheim und ging bei der Zerstörung des Schloßmuseums im Zweiten Weltkrieg verloren. DOMASZEWSKI gibt die unvollständige Inschrift des Steins aufgrund der Angaben ZANGEMEISTERs (,Zangemeister contulit.") im CIL wie folgt wieder. ${ }^{50}$

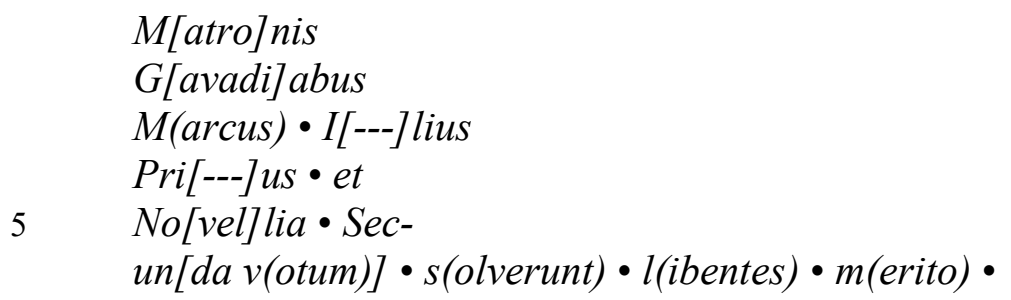

\footnotetext{
${ }^{41}$ Auf einem Meilenstein aus Den Haag muss laut AE 2000, 1022 = EDH Nr.HD044898 die Dativform T(ito) Aeli(o) H[ad]r(iano) gelesen werden. Eine neuere Lesung bietet jedoch T. Ael(io) H[ad]r(iano) (AE 2003, 1229).

${ }^{42}$ Den Namen Marinius Cossus verzeichnet zu Recht auch der Index des CIL: CIL XIII/5 (Index) 14.

S. ferner SCHARF 1938, 93 [,Atti(lius) ..., Sohn des Marin(i)us Cossus“].

${ }^{43}$ CIL XIII 8787 = LAZZARO 1993, 262. Nr.308.

${ }^{44}$ CIL XIII 7119 = LAZZARO 1993, 204-205. Nr.220.

${ }^{45}$ CIL XIII 7958/59 (Euskirchen-Rheder).

${ }^{46}$ CIL XIII 8003a $=$ LEHNER 1918, 192. Nr.509 (Bonn-Endenich).

${ }^{47}$ 1. N. 205. Nr.345 = AE 1927, 66 (Stuttgart-Bad Cannstatt).

${ }^{48}$ S. hierzu A. KAKOSChKE, FeRA 26 (2015), 14-20.

${ }^{49}$ CIL XIII 7885-7886, 7888-7893.

${ }^{50}$ CIL XIII 7887. Bezüglich der Namen s. auch CIL XIII/5 9, 15 (Index).
} 


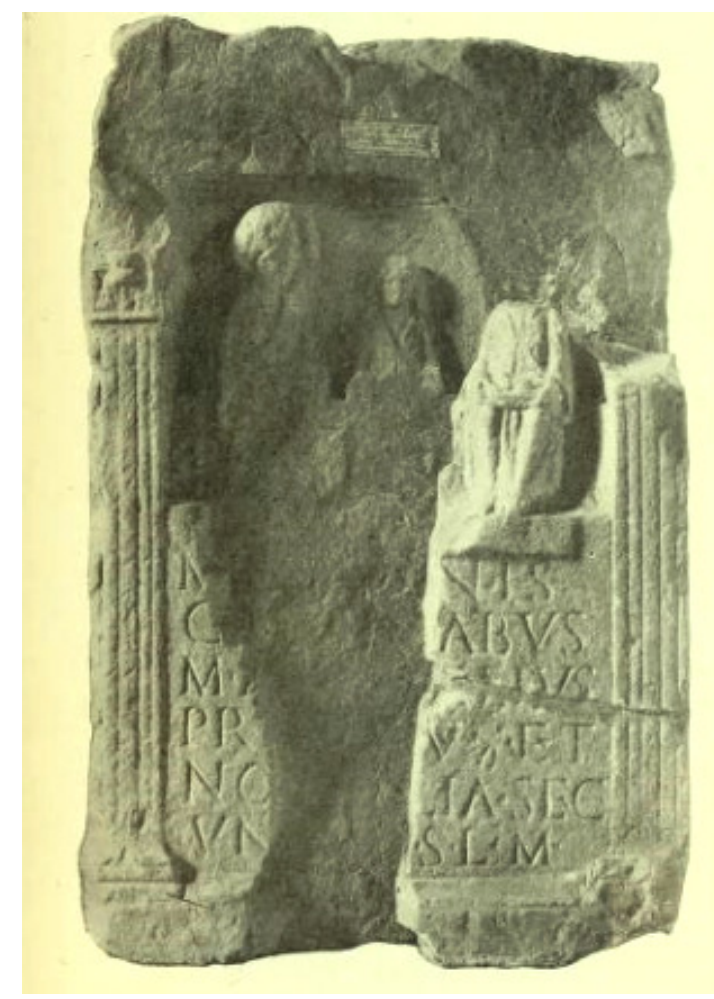

Abb.4a: Weihinschrift aus Titz-Rödingen: CIL XIII 7887
(nach ÉSPERANDIEU 1922, 288-289. Nr.6344)

Diese Lesung wird bis heute zitiert, ${ }^{51}$ obwohl HAUG bereits im Jahre 1877 eine vielleicht überzeugendere Ergänzung der Inschrift vorgelegte, die auch von einigen späteren Bearbeitern der Inschrift übernommen wurde. ${ }^{52}$ Gemäß HAUG lautet der Text wie folgt: ${ }^{53}$

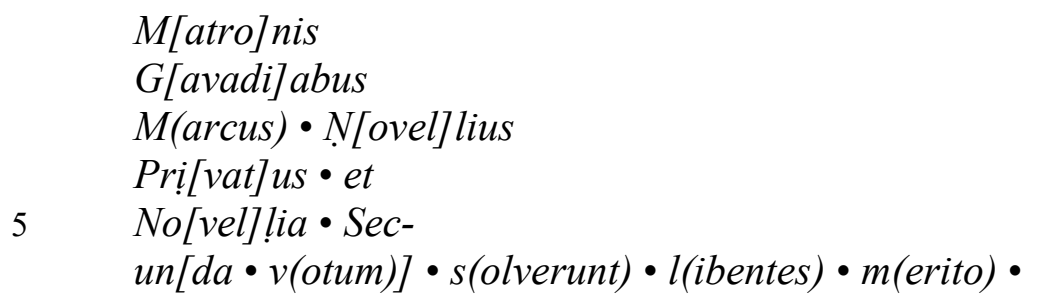

Wie eine Rekonstruktionszeichnung der Inschrift (Abb.4b) auf der Grundlage einer Vorkriegsaufnahme (Abb.4a) zeigt, ${ }^{54}$ ist die Lesung HAUGs durchaus überzeugend, wenngleich die Laufweite der ergänzten Buchstaben im Namen NOVELLIVS recht groß erscheint. HAUG bemerkt zu seiner Lesung: „Die Ergänzung der Namen Z. 3-5

\footnotetext{
51 Weisgerber 1968, 27. Nr.112, SpickermanN 1994, 346. Nr.2, KAKoschKe 2006, 450. Nr.100, SCHEID 2006, 321, GARMAN 2008, 152. Nr.311.

52 RIESE 1914, 330. Nr.3147, GUTENBRUNNER 1936, 215. Nr.56-5.

${ }^{53}$ HAUG 1877, 28. Nr.25. Die Lesung HAUGs wurde hier in eine heute gängige Umschrift übertragen.

${ }^{54}$ Das bei ÉSPERANDIEU 1922, 288-289. Nr.6344 gebotene Photo verdankt der Autor H. GROPENGIEßER, dem damaligen Kustos des Großherzoglichen Hofantiquariums in Mannheim. Das Photo ist offensichtlich identisch mit der Abbildung bei M. PERSE, Jülicher Geschichtsblätter 67/68 (1999/2000), 887. Abb.3. Die Rechte für dieses Photo liegen laut PERSE bei den Reiss-Engelhorn Museen (Mannheim). Nach freundlicher Auskunft von Frau L. REIBLICH verfügen die Reiss-Engelhorn Museen jedoch über keine alten Aufnahmen der Inschrift.
} 
ist zwar nicht ganz sicher, aber nach der Distanz und den Buchstabenresten wahrscheinlich ...".

Die auch von DOMASZEWSKI übernommene Ergänzung des Namens in Zeile 5 zum gängigen Gentiliz Novellia, das auch in der Germania inferior noch zweimal bezeugt ist ${ }^{55}$ erscheint aufgrund fehlender Alternativen sicher. ${ }^{56}$ Zwar findet sich im OPEL die Ergänzung zu Nobilius, ${ }^{57}$ doch ist diese Lesung aufgrund der Kürze des Namens nicht möglich.

Von dem Gentiliz in Zeile 3 ist am Anfang gemäß HAUG und den Angaben im CIL nur der untere Teil einer Vertikalhaste erhalten. Es folgt dann eine Lücke von vier bis fünf Buchstaben. Der Name endet ebenso wie das Gentiliz in Zeile 5 auf -lius. Die von ÉSPERANDIEU erwogene Ergänzung zu I[u]lius ist demnach nicht denkbar. ${ }^{58}$ Durchaus möglich und aufgrund des Namens in Zeile 5 naheliegend erscheint dagegen die Ergänzung zu Novellius, wenngleich selbstverständlich auch noch andere Namen in Betracht kommen. ${ }^{59}$

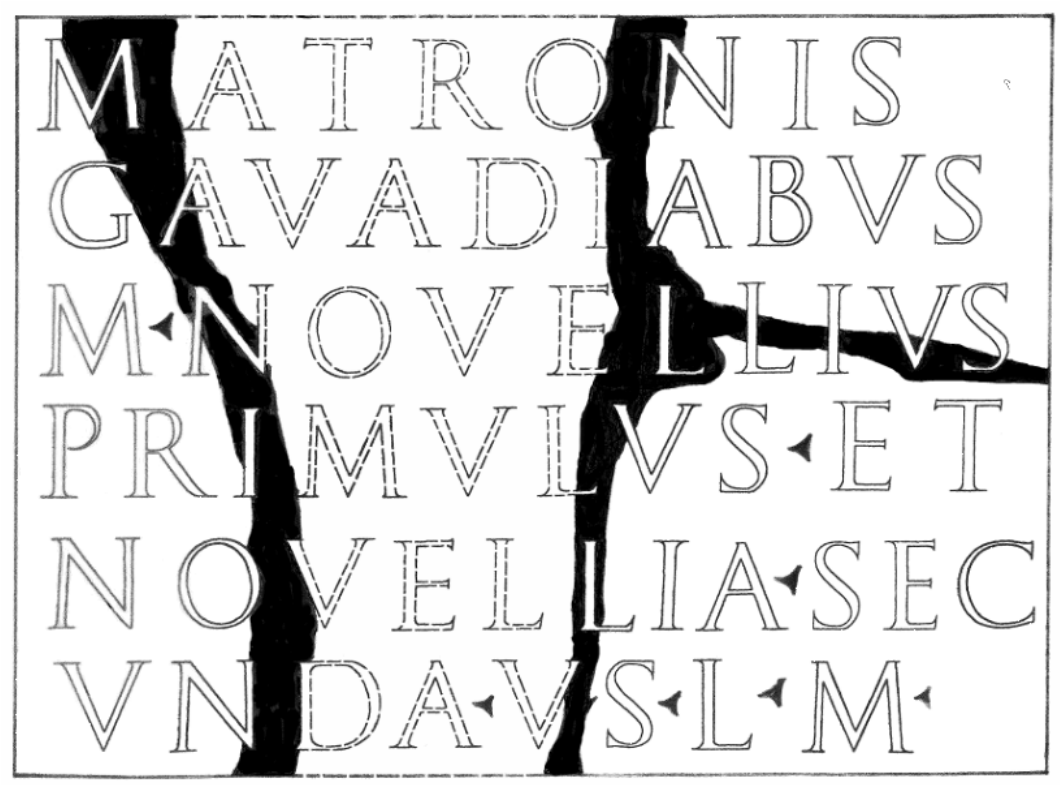

Abb.4b: Leicht idealisierte Rekonstruktionszeichnung der Weihinschrift CIL XIII 7887 aus Titz-Rödingen (Zeichnung A.K.)

Noch vor HAUg las BeCKer in Zeile 3 das Gentiliz A[em]ilius. ${ }^{60}$ Diese Lesung, die von IHM ohne Vorbehalte bestätigt wurde, ${ }^{61}$ ist insofern interessant, als der noch erhaltene Teil der Vertikalhaste des zweiten Buchstabens in Zeile 3 offenbar tatsächlich eine Neigung nach rechts aufzuweist. Zwar mag ein (altes) Photo täuschen, doch könnte es sich bei dem ersten Buchstaben des Gentilnomens um ein A handeln. Auf

\footnotetext{
${ }^{55}$ CIL XIII $8408=$ IKöln $^{2}$ 413. Nr.518 (Köln/CCAA), 2. N. 101-102. Nr.179 (Bonn/Bonna). Zum Namen s. KAKOSCHKE 2006, 288. Nr.867.

${ }^{56}$ Die Ergänzung bietet vor HAUG bereits J. BECKER, BJb 42 (1867), 107. Nr.6.

${ }^{57}$ OPEL III 103. Vgl. auch KAKOSCHKE 2006, 286. GN 859. Das OPEL III 105 bietet aber auch die Lesung No[vel]lia.

${ }^{58}$ ÉSPERANDIEU 1922, 288-289. Nr.6344. Eine entsprechende Ergänzung bieten auch RöSGER 1996, 13 und die EDCS Nr.11100112.

${ }^{59}$ Aus dem Bestand der in den zwei germanischen Provinzen bezeugten Namen sei hier auf folgende Namen verwiesen: Hostilius, Nasellius, Pompilius, Primulius, Proculius, Publilius.

${ }^{60}$ J. BECKER, BJb 42 (1867), 107. Nr.6.

${ }^{61}$ M. IHM, BJb 83 (1887), 149-150. Nr.301 („Die Ergänzungen sind sicher.“).
} 
einer Vergrößerung des Photos (Abb.5a) scheint sogar der linke Ansatz der Horizontalen noch erkennbar zu sein. ${ }^{62}$ Eine Ergänzung zum gängigen Gentiliz Aemilius ist jedoch nicht möglich, da die ergänzten Buchstaben den Platz in der Lücke nicht ganz ausfüllen. In Frage kommen aber einige weitere Namen. Von den in den germanischen Provinzen bezeugten Namen sind Agisilius und Amabilius denkbar, ferner auch Anaillius, sofern man mit HAUG und DOMASZEWSKI am Ende von Zeile 3 lediglich LIVS entziffert. ${ }^{63}$ Aufgrund der Buchstabenbreite mag man sich für eine Ergänzung zum Pseudogentiliz Amabilius entscheiden (Abb.5b). Da jedoch zu viele Buchstaben schon bei der Auffindung der Inschrift verloren waren und sich die Lesung der noch vorhandenen Buchstaben nicht mehr am antiken Objekt überprüfen lässt, muss die Lesung selbstverständlich spekulativ bleiben.

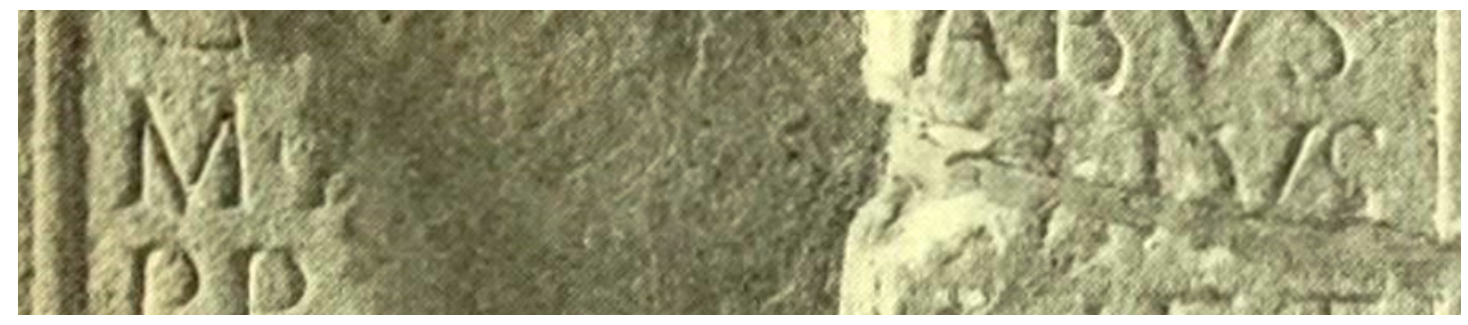

Abb.5a: Weihinschrift aus Titz-Rödingen (Detail): CIL XIII 7887 (nach ÉSPERANDIEU 1922, 288-289. Nr.6344)

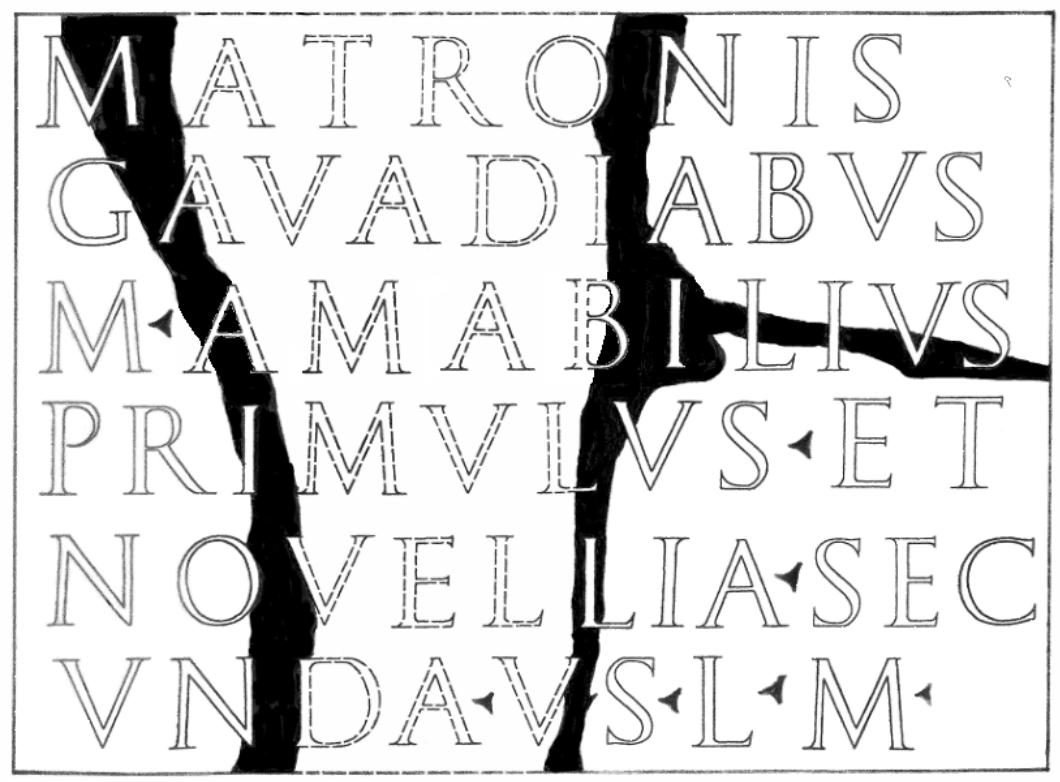

Abb.5b: Leicht idealisierte Rekonstruktionszeichnung der Weihinschrift CIL XIII 7887 aus Titz-Rödingen (Zeichnung A.K.)

\footnotetext{
${ }^{62}$ Irritierenderweise gibt J. BECKER, BJb 42 (1867), 107. Nr.6 trotz seiner im Kommentar zur Inschrift vorgeschlagenen Lesung A[em]ilius den Anfang von Zeile 3 mit den Buchstaben MI (ohne Worttrenner) wieder und stellt im Kommentar zur Inschrift fest, dass „der Strich hinter M senkrecht ist.“

${ }^{63} \mathrm{Zu}$ Agisilius s. KaKoschKe 2006, 64. GN 36, OPEL I² 35. - Zu Amabilius s. KaKoschKe 2006, 69. GN 60, OPEL I² 45. - Zu Anaillius s. KAKOSCHKE 2006, 72-73. GN 76, OPEL I² 51.
}

http://www.fera-journal.eu 
Das Cognomen in Zeile 5/6 lässt sich wiederum sicher zum gängigen Secunda ergänzen. ${ }^{64}$ Dagegen bieten sich für die Ergänzung des Cognomens in Zeile 4 mindestens zwei Möglichkeiten an. Neben der von HAUG favorisierten Lesung Pri[vat]us könnte hier auch Pri[mul]us gelesen werden. ${ }^{65}$

\section{V.}

In der Chronik des Registrators JoHANNES TURCK (1550-1625) findet sich die Zeichnung eines Grabsteins, der Anfang des 17. Jahrhunderts in Xanten (an einem unbekannten Ort) entdeckt wurde (Abb.6). Das Fundstück gelangte zunächst in die Antikensammlung auf Schloss Wissen, die von Graf Wessel von Loe, dem Herrn zu Wissen (1574-1629), in den Jahren nach 1591 angelegt wurde. Der Stein, dessen Maße und Material unbekannt sind, gilt heute als verschollen. DIEDENHOFEN gibt die Inschrift aufgrund der überlieferten Zeichnung wie folgt wieder: ${ }^{66}$

$\begin{array}{ll} & \text { Have Calventi Calv- } \\ & \text { entius te resalutat } \\ & \text { C(aius) Calventius Omvi- } \\ & \text { ifil(ius) Ouf(entina tribu) Med(iolano) hic situs } \\ 5 & \text { est an(norum) XLIIX mil(es) leg(ionis) V } \\ & \text { [st]ip(endiorum) XXIIX et coniugi et } \\ & \text { [fili]o frater pro pietate } \\ & \text { [flete] coaequales mors haec } \\ & \text { [mea fletibu]s apta est vi[vi]t[e] fel[i]ces qu[ibus est] } \\ & \text { [p]ar patria dulcis } \\ & \text { [her]es e sua [pec(unia) e(rexit)] }\end{array}$

Im Fokus steht im Folgenden der Name bzw. die Namensform des verstorbenen Legionärs der legio $V$ (Alaudae) aus dem oberitalischen Mailand/Mediolanum. Auf der Zeichnung TuRCKs liest man C. Calventius Omvii fil(ius). Demnach trug der Legionär einen Namen der Form „Pränomen + Gentiliz + cognominale Filiation“. Diese charakteristische Namensform tritt bis in das erste Jahrhundert $\mathrm{n}$. Chr. vor allem in Oberitalien auf, der Heimat des Legionärs. ${ }^{67}$ Zudem ist die Namensform durch Personen aus Oberitalien in der Fremde bezeugt. So finden sich in Mainz/ Mogontiacum zwei Legionäre aus Ivrea/Eporedia und Este/Ateste, namens [---] Virius Macconis f. Pol. ${ }^{68}$ und [---] Atinius Sept<i? $>$ mi f. Romilia (Abb.7). ${ }^{69}$ Durch einen

\footnotetext{
${ }^{64}$ Zum Namen s. KAKOSCHKE 2008, 306-309. Nr.2757, OPEL IV 59-61.

${ }^{65}$ Eine entsprechende Lesung bietet auch ÉSPERANDIEU 1922, 288-289. Nr.6344. - Zum gängigen Privatus s. KAKOSCHKE 2008, 226. Nr.2461, OPEL III 164. - Zum gängigen Primulus s. KAKOSCHKE 2008, 219-220. Nr.2444, OPEL III 160-161.

${ }^{66}$ DiEDENHOFEN 1974, 162-163. Nr.18 (mit Zeichnung TURCKs). Auf der Zeichnung TURCKs basieren alle anderen Wiedergaben der Inschrift, so auch CIL XIII 8644.

${ }^{67}$ S. z.B. CIL V 774 (Aquileia/Aquileia; Sex. Baebius Bai f.), 2019 (Oderzo/Opitergium; M.' Terentius Voltietis f.), 4049 (Medole; P. Catius Callavi f.), 4153 (Scarpizzolo; P. Mucius Biraci f.), 4281 [Brescia/Brixia; M. et C. Valeri(i) Senecionis fili(i)], 4926 (Ponte Zanano; C. Valerius Secundini f.), 4960 (Cividate Camuno; C. Claudius Sassi f. Quir.), 5176 (Bergamo/Bergomum; C. Statius Cirusi f.), 5817 (Mailand/Mediolanum; [L. Aebuti]us Lucumonis [f.]), 6862 (Roisan; C. Iulius Mami fil.), 7013 (Torini; Augusta Taurinorum; C. Aebutius Rufi f. Stel.), 7351 (Casteggio/Clastidium; T. Catius Terti f.). Bei den genannten Vaternamen kann es sich in einigen Fällen ebenso um ausgeschriebene oberitalische Pränomina handeln. $\mathrm{Zu}$ diesen s.u.

${ }^{68}$ CIL XIII 6914 = LUPA Nr.25307 (mit Photo).

${ }^{69}$ CIL XIII 7244. - Ob die Namensform auch durch einen Silberring aus Frankfurt Heddernheim bezeugt ist, bleibt fraglich. Der Name auf dem Ring lautet (offenbar) T. Carm(inius?) Scraini (f.?) (4. N.
} 
Zufall sind beide Grabinschriften nur fragmentarisch überliefert. Bei beiden Steinen fehlt (u.a.) der obere Teil des Steins und somit die erste Zeile der Inschrift, in der aufgrund der Namensform zwingend ein Pränomen zu ergänzen ist. Für die Inschrift CIL XIII 7244 gibt ZANGEMEISTER im Kommentar zur Inschrift einen entsprechenden Hinweis: „Praenomen supra gentilicium positum erat, ut n. 6882. 6895. 6905.“70 Bei dem Pränomen muss es sich jedoch nicht zwingend um ein klassisches lateinisches Pränomen gehandelt haben, das wie in den von ZANGEMEISTER genannten Inschriften in abgekürzter Form zentriert in der ersten Zeile stand. Vielmehr kann hier auch ein ausgeschriebenes oberitalisches Pränomen wie Primus, Rufus, Secundus oder Tertius gestanden haben. ${ }^{71} \mathrm{Zu}$ beachten ist auch, dass der Erbe des [---] Virius Macconis $f$. Pol. den Namen Firmus Val[erius --- f.] trug und somit ein oberitalisches Pränomen führte. ${ }^{72}$

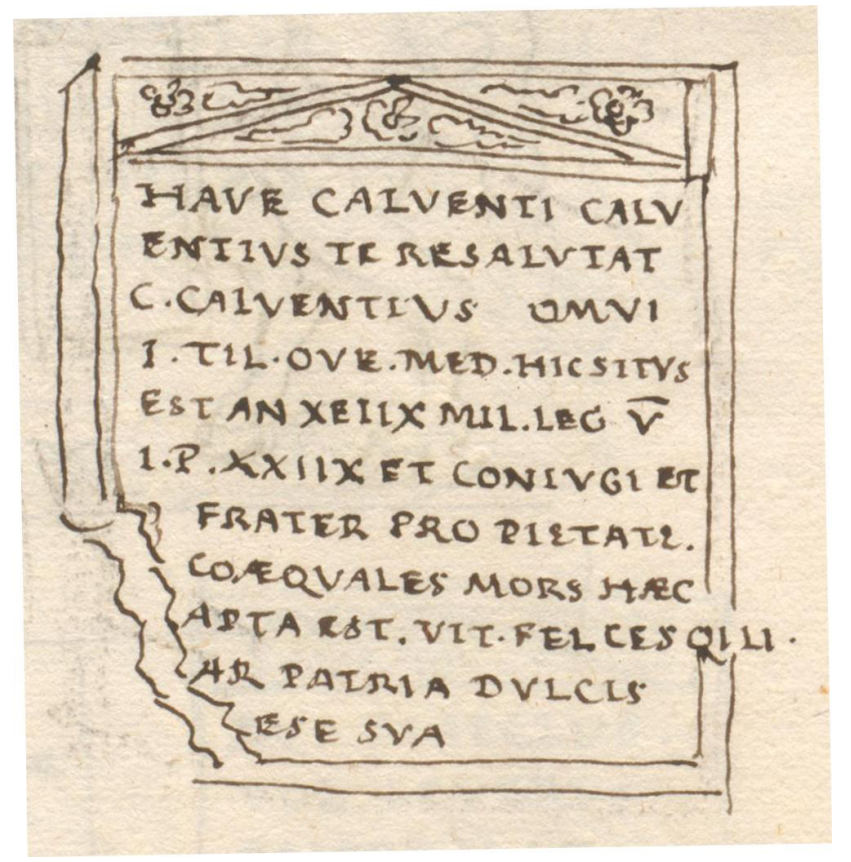

Abb.6: Grabinschrift aus Xanten: CIL XIII 8644

(nach Chronik des Johannes Turck HStA Düsseldorf HS C III 9, f. 81 ${ }^{\text {v }}$

[C Landesarchiv NRW, Abteilung Rheinland (heinland@lav.nrw.de)]

515-516. Nr.116). Auf jeden Fall finden sich die meisten Belege für das Gentiliz Carminius in Oberitalien (KAKOSCHKE 2006, 127-128. GN 284).

${ }^{70}$ Dennoch geben folgende Bearbeiter den Namen in der Inschrift CIL XIII 7244 fälschlicherweise ohne Pränomen wieder: RIESE 1914, 128. Nr.1136, KRONEMEYER 1983, 80, HOPE 2001, 163, KAKOSCHKE 2006, 90. GN 146/2. Die korrekte Lesung bieten: F. BosCOLO, Erga-Logoi 4/2 (2016) 208-209. Nr.27, EDCS 11001332. - Für die Inschrift CIL XIII 6914 fehlt ein Hinweis zum fehlenden Pränomen im Kommentar zur Inschrift. Fehlerhafterweise ohne Pränomen geben den Namen (daher) folgende Bearbeiter/Datenbanken wieder: RIESE 1914, 112. Nr.962, KRONEMEYER 1983, 82, KAKOSCHKE 2006, 440-441. GN 1452/7, EDCS Nr.11000969, LUPA Nr.25307 (mit Photo), EDH Nr.056137. Korrekt ist die Wiedergabe bei: Hope 2001, 152.

${ }^{71}$ Entsprechende Pränomina sind durch Legionäre aus Oberitalien auch in Mainz/Mogontiacum bezeugt. S. z.B. CIL XIII 6885 (Primus Aebutius L. f. Veturia), 6899 [Secundus Metilius M. f. St(e)1.], 6939 [Ter(tius) Exsomnus Ter(tii) f. Ani.], 3. N. 185. Nr.166 [Ruf(us) Valerius Q. f. Serg.]. Zu den ungewöhnlichen Pränomina s. H. INSTINSKY, JbRGZM 5 (1958), 239-245.

${ }^{72}$ Die entsprechende Namensform mit einem ausgeschriebenen einheimischen Pränomen ist in Oberitalien ebenfalls mehrfach bezeugt: S. z.B. CIL V 6789 (Ivrea/Eporedia; Firmus Cliccius Nasonis f.), 7064 (Torino/Augusta Taurinorum; Rufus Atilius Licini f.), 7656 (Centallo; Mocus Caranius Nevi f. Pol.), 7728 [Bene Vagienna/Augusta Bagiennorum; Firmus Lucani(us) Gemini f. Cam.], AE 1975, 434 (Mezzane; Rufus Acutius Comincilonis [f.]). 


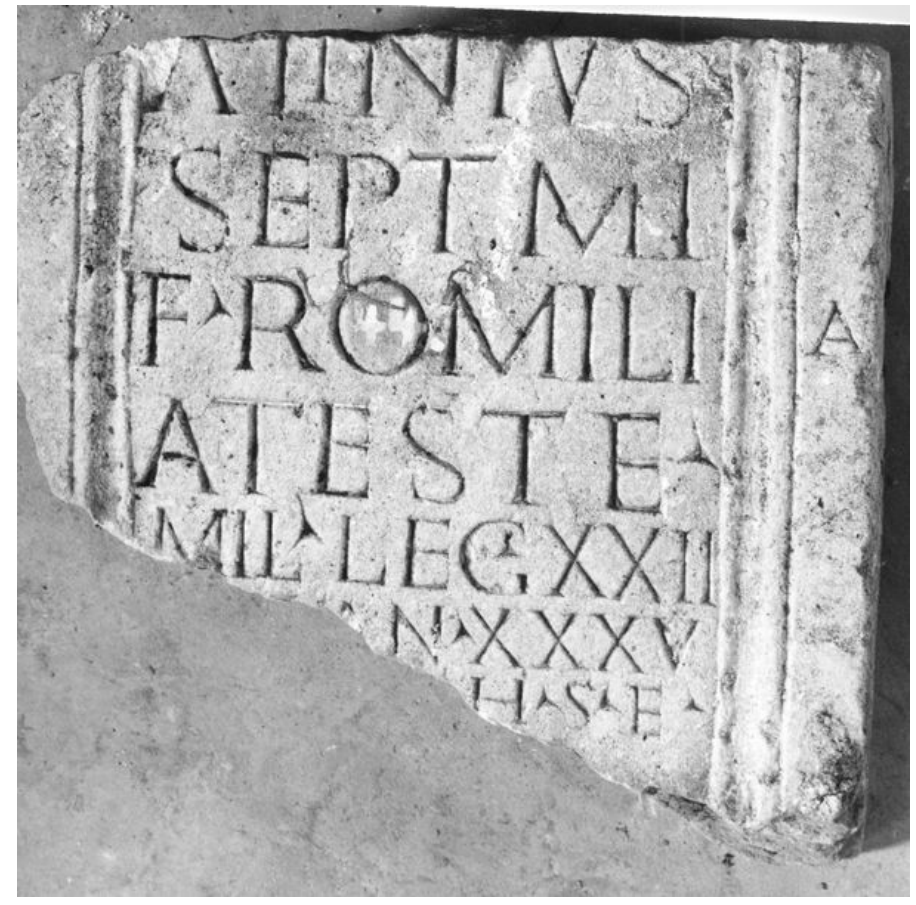

Abb.7: Grabinschrift aus Mainz-Hechtsheim/Mogontiacum: CIL XIII 7244 (C) CIL XIII/2-Projekt, Universitäten Trier/Flensburg)

Das Gentiliz des Legionärs aus Xanten, Calventius, ist überall bezeugt. Die meisten Belege stammen jedoch aus Oberitalien. ${ }^{73}$ Neben der Namensform bestätigt somit auch das Gentilnomen eine Herkunft des Legionärs aus der Gallia cisalpina.

Unsicher bleibt der überlieferte Name des Vaters. Bei dem von TURCK gelesenen OMVII wird es sich höchstwahrscheinlich um einen Fehler handeln. Ein entsprechender Name ist jedenfalls anderweitig nicht bezeugt. Die in den Stein geschlagenen Buchstaben waren möglicherweise nicht mehr eindeutig zu entziffern. Bleibt man möglichst nah bei den von TURCK angegebenen Buchstaben/Hasten bietet sich RIESE folgend die Lesung $(H) o m u<l>i$ oder $(H)$ om $u<l>(l) i$ an. ${ }^{74}$ Wenn der Vater des Calventius nicht einen einheimischen keltischen Namen trug, dann führte er also vielleicht den relativ gängigen lateinischen Namen Homullus, der auch vielfach in der Form Omullus bezeugt ist. ${ }^{75}$

Der Grabstein aus Xanten muss der Zeit von 10 bis $69 \mathrm{n}$. Chr. angehören, in der die legio V Alaudae in Xanten/Vetera stationiert war. ${ }^{76}$ Da C. Calventius noch kein Cognomen trug, datieren DiEDENHOFEN und SCHALLES den Grabstein in die vorclaudische Zeit, ${ }^{77}$,in die Jahre vor $40 \mathrm{n}$. Chr.“78 Diese Datierung ist zumindest fraglich, da ein genauer Zeitpunkt für die Änderung in der Namensgebung römischer Legionäre nicht genannt werden kann. Nach allgemeiner Auffassung führen die

\footnotetext{
${ }^{73}$ Zum Namen s. KAKOSCHKE 2006, 119. GN 249, OPEL II 26. - Angesichts der vielen Belege für den Namen ist die Vermutung von Schalles 2006, 114, dass der auf dem Grabstein CIL XIII 8644 namentlich nicht genannte Bruder des C. Calventius mit einem T. Calventius, dem Besitzer eines Infanteriehelms aus Kalkar-Hönnepel identisch ist, recht gewagt.

${ }^{74}$ RIESE 1914, 86. Nr.703. S. auch schon CLE II/1. 175. Nr.374. Vgl. dagegen BRAMBACH 1867, 59. Nr.218, der [R]omuli lesen möchte. Ob vor dem zu lesenden OMVI jedoch noch ein Buchstabe fehlt, wie es die Lücke auf der Zeichnung andeutet, bleibt fraglich.

${ }^{75}$ Zum Namen s. KAKOSCHKE 2007, 410. CN 1533, OPEL II 184 (jeweils mit dem Beleg CIL XIII 8644).

${ }^{76}$ SCHALLES 2006, 113.

77 DIEDENHOFEN 1974, 163, SCHALles 2006, 113.

${ }^{78}$ SCHALLES 2006, 114.
} 
Legionäre „seit claudischer Zeit so gut wie regelmäßig“ ein Cognomen. ${ }^{79}$ Der Grabstein des zuvor genannten [--] Atinius Sept $<i$ ? > mi f. Romilia (Abb.7) muss jedoch auf jeden Fall der Zeit nach $43 \mathrm{n}$. Chr. angehören, da der Legionär in der legio XXII Primigenia diente. ${ }^{80}$ So mag vielleicht auch der Grabstein des Calventius einer etwas späteren Zeit zuzurechnen sein. Gegen diese Auffassung spricht auch nicht die Form der Stele mit Horizonalabschluss und Reliefgiebel, da vergleichbare Grabsteine auch noch aus der flavischen Zeit bekannt sind. ${ }^{81}$

\section{VI.}

In der Kirche St. Ursula, in Köln/CCAA, entdeckte man im Jahre 1967 einen Inschriftensockel (Höhe 23,5 cm - Breite $26 \mathrm{~cm}$ - Tiefe 17,3 cm) aus Kalkstein (Abb.8). Der fragmentarische mehrfach gebrochene Stein, der in Zweitverwendung als Ecke eines Stützfundamentes fungierte, musste vollständig restauriert werden. Dabei wurde der Unterblock völlig ergänzt. B. und H. GALSTERER lesen die unvollständige Inschrift des Steins, den sie mit einem Fragezeichen dem zweiten Jahrhundert n. Chr. zuordnen, wie folgt: ${ }^{82}$

$$
\begin{aligned}
& \text { [Genio?] contiti } \\
& \text { uno[...] rum } \\
& \text { [---ca. 5-6---]norum } \\
& M(\text { arcus }) \cdot C a p i[t] o(n i u s ?) \text { Vitalis } \\
& 5 \quad \underline{\text { et }} \cdot M(\text { arcus }) \underline{\text { Temi[n] }} \text { (ius?) } \bullet \text { Aman- } \\
& \text { dus cu[r]ator(es) d(ono) d(ederunt) }
\end{aligned}
$$

Der Text lässt sich offensichtlich nicht vollständig ergänzen. Dennoch können m.E. kleine Korrekturen angemerkt werden: Der von B. und H. GALSTERER in Zeile 4 gelesene Name wurde offenbar nicht korrekt ergänzt bzw. gelesen. Die abgekürzte Form Capito(nius) ist ohnehin recht auffällig. ${ }^{83}$ Vor allem aber handelt es sich bei dem von B. und H. GALSTERER gelesenen $\mathrm{O}$ vor VITALIS recht eindeutig um ein D. $\mathrm{Da}$ die auf das $\mathrm{C}$ folgenden Buchstaben sich m.E. einer sicherern Lesung entziehen, liegt die Lesung C[lau]d(ius) nahe. Allerdings scheint die Lücke zwischen $\mathrm{C}$ und D für die ergänzte Buchstabengruppe LAV etwas zu groß zu sein. Wenn es sich bei dem Buchstaben nach dem $C$ tatsächlich um ein A handeln sollte, könnte man ebenso die Lesung $C a[n d i] d(i u s)$ bzw. Cạ[ndi]d(ius) in Erwägung ziehen. Eine größere Sicherheit ist angesichts des Erhaltungszustandes der Inschrift jedoch nur schwer zu gewinnen.

\footnotetext{
${ }^{79}$ BOPPERT 1992, 33 (mit weiteren Literaturhinweisen). S. ferner KRONEMYER 1983, 18.

${ }^{80}$ Zum Zeitpunkt der Stationierung der legio XXII Primigenia in Mainz/Mogontiacum s. KRONEMAYER 1983, 12-13, BOPPERT 1992, 30.

${ }^{81}$ S. z.B. CIL XIII 6831 = BOPPERT 1992, 255-256. Nr.150. Taf.106 (Zeichnung), $6895=$ BopPERT 1992, 258-259. Nr.153. Taf.106 (Zeichnung), 4. N. 491. Nr.62 = BOPPERT 1992, 256-257. Nr.151.

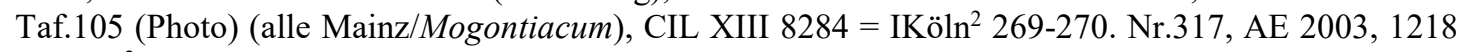
$=$ IKöln ${ }^{2}$ 312-313. Nr.375 (beide Köln/CCAA).

${ }^{82}$ IKöln $^{2}$ 63. Nr.42 (mit Photo) = EDCS Nr.00500007 (mit identischer Lesung). Nicht gekennzeichnete Ligaturen wurden hier vom Autor ergänzt. - Der Sockel befindet sich heute im Römisch-Germanischen Museum in Köln (Inv.-Nr.67,2154).

${ }^{83}$ Zum Namen s. KAKOSCHKE 2006, 124-125. GN 271, OPEL II 33-34 (jeweils mit dem vermeintlichen Beleg aus Köln/CCAA).
} 


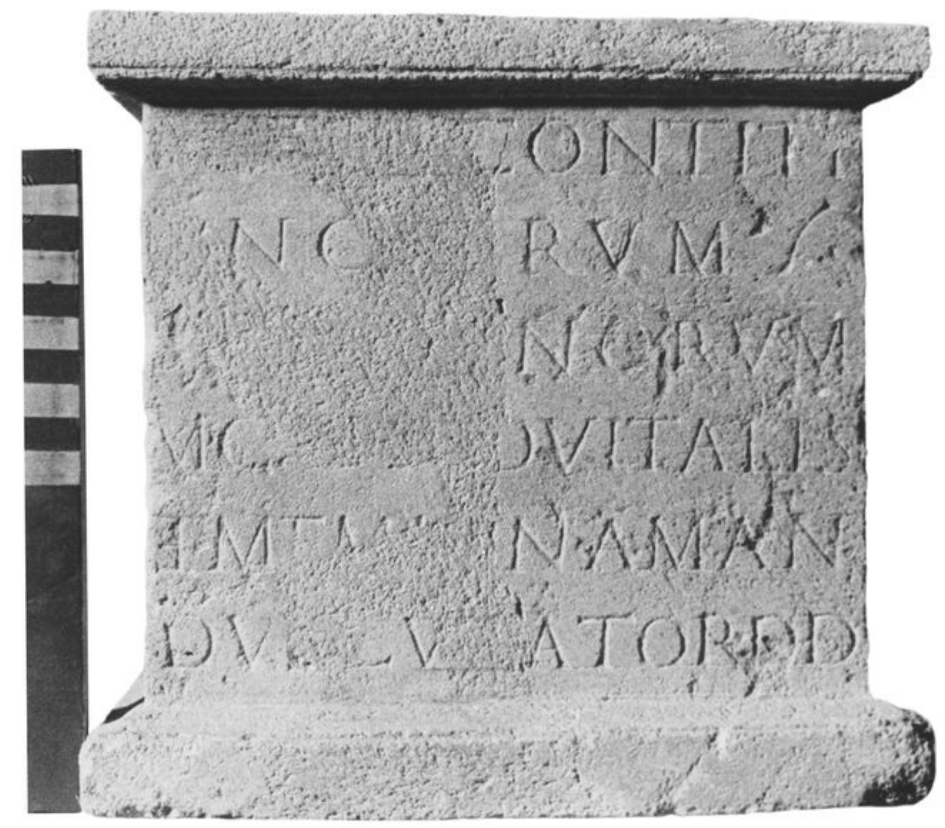

Abb.8: Fragmentarische Weihinschrift aus Köln/CCAA: AE 1974, 444 (C) Römisch-Germanisches Museum, Köln)

Ferner kann die Lesung des Gentilnomens in Zeile 5, des nicht weiter bezeugten Namens Teminnius, m.E. nicht stimmen. Die von B. und H. GALSTERER entzifferte zweite ET-Ligatur scheint nicht möglich, da der Platz für ein $\mathrm{T}$ zum links angrenzenden $\mathrm{M}$ viel zu schmal ist. Auch ist zwischen dem vermeintlichen Pränomen und der vermeintlichen ET-Ligatur kein Worttrenner zu erkennen, so dass die Lesung $M$ (arcus) wohl eher ausgeschlossen werden sollte. In zwei älteren Publikationen lesen B. UND H. GALSTERER in Zeile 5 den Namen Mem[no]n(ius) bzw. Memn[o]n(ius). ${ }^{84}$ In der vorliegenden Rekonstruktion der Inschrift reicht der Platz für die Buchstaben NO in der vorhandenen Lücke jedoch eindeutig nicht aus. Letztendlich lässt sich somit lediglich der Name Mem[... n(ius?) entziffern. Eine Ergänzung des Gentilnomens ist aufgrund des bisher bekannten Namenmaterials (noch) nicht möglich.

Schließlich muss man in dem von B. und H. GALSTERER in Zeile 2 entzifferten $\mathrm{O}$ ein $\mathrm{C}$ erkennen. Die Buchstabengruppe VNC ergibt allerdings wenig Sinn. Eine sinnvolle Ergänzung der ersten Zeilen ist somit weiterhin nicht möglich.

\section{VII.}

In der Kapelle der Kirche von Langensoultzbach (dép. Bas-Rhin) fand sich im Jahre 1961 eine fragmentarische Grabinschrift (Höhe $68 \mathrm{~cm}$ - Breite $52 \mathrm{~cm}$ - Tiefe $9 \mathrm{~cm}$ ). Die dünne Tafel aus rötlichem Sandstein, die in der Kirche als Bodenplatte fungierte, ist an allen Seiten abgeschlagen bzw. bestoßen (Abb.9). WUILLEUMIER, der Ersteditor der Inschrift, gibt folgende Lesung des etwas nachlässig eingeschlagenen Textes: ${ }^{85}$

\footnotetext{
${ }^{84}$ B. u. H. GAlsterer, KJb 13 (1973), 92-93. Nr.2. Abb.1 (Zeichnung), Taf.32/2 (Photo), GALSTERER 1975, 18-19. Nr.32. Taf.8 (Photo). S. auch AE 1974, 444 und EDH Nr.HD011181. Zum Namen s. auch KAKOSCHKE 2006, 270. GN 785, OPEL III 75 (jeweils mit dem vermeintlichen Beleg aus Köln/ CCAA).

${ }^{85}$ ILTG 176-177. Nr.436. - Der Stein befindet sich heute in der zu einem Museum umgestalteten Kapelle der protestantischen Kirche von Langensoultzbach (Musée de Langensoultzbach, Chapelle de l'Église).
} 

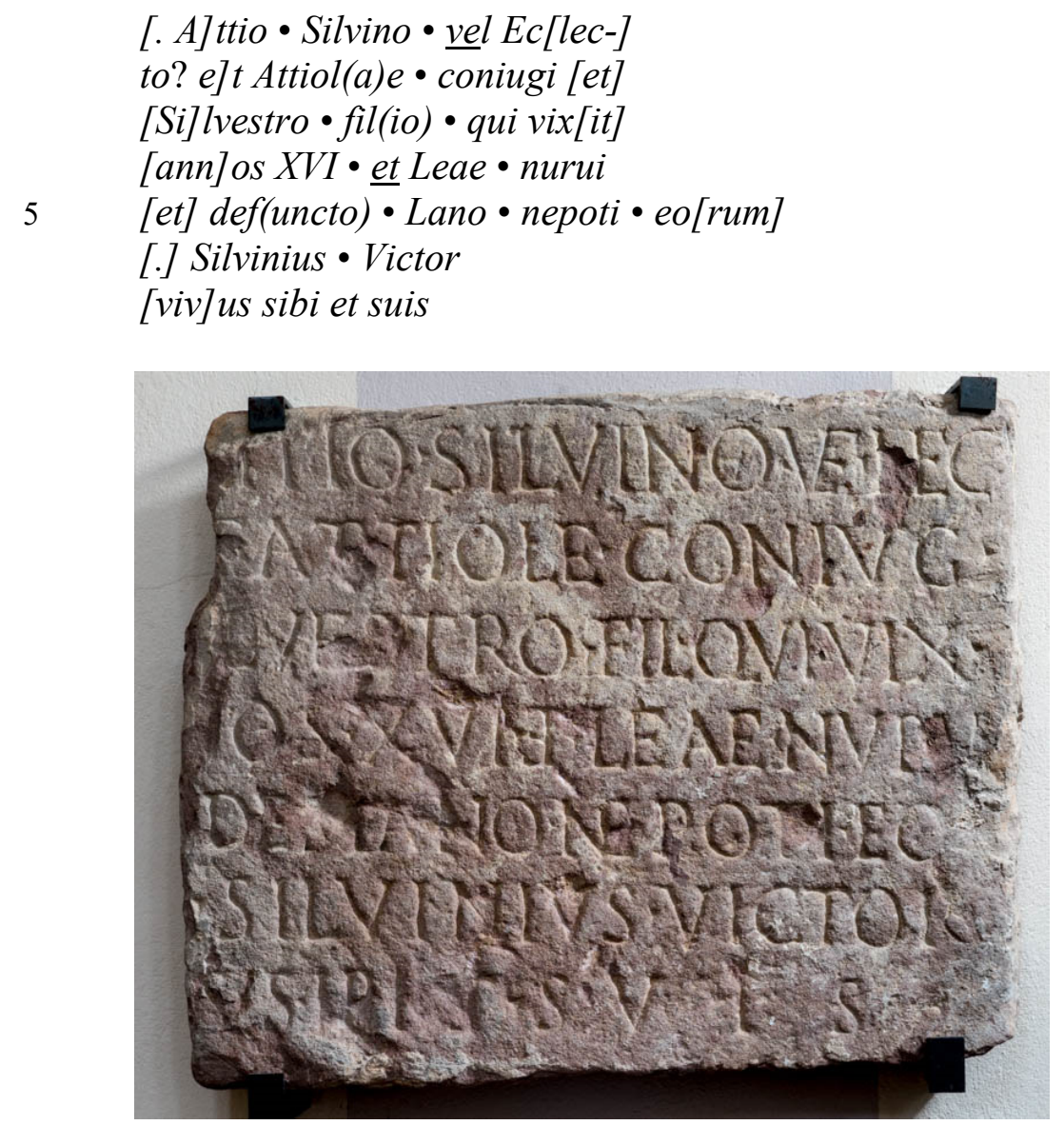

Abb.9: Fragmentarische Grabinschrift aus Langensoultzbach: ILTG 436 [C Ortolf Harl, Wien (LUPA)]

Obwohl diese Lesung Zweifel wecken sollte, folgen alle weiteren Bearbeiter der Inschrift der Textwiedergabe WuILleuMIERs. ${ }^{86}$ Fraglich erscheint vor allem die Lesung am Ende der ersten Zeile. WuILlEuMIER zufolge führt der Verstorbene den Namen [. A]ttius Silvinus vel Ec[lectus]. Demnach trug der Mann neben seinem Cognomen Silvinus einen weiteren Namen, Eclectus, der mit vel eingeleitet wird. Entsprechende Supernomina finden sich relativ häufig im Namenmaterial. Diese Namen werden allerdings entweder mit qui/quae et bzw. sive (agnomina) oder mit signum (signa) eingeleitet. ${ }^{87}$ Der Umstand, dass sich im gesamten epigraphischen Material kein mit vel eingeleiteter zusätzlicher Name findet, spricht nicht für die vorgelegte Lesung vel Ec[lecto]. ${ }^{88}$ Der epigraphische Befund scheint den Zweifel zu erhärten. Offenbar lässt sich zwischen dem ligierten VE und LEC noch ein Worttrenner ausmachen. Da es sich bei dem gelesen $C$ ebenso um ein $G$ handeln kann, liegt die Lesung ve(terano) leg(ionis) nahe. Aufgrund der geographischen Lage des Fundortes, im Gebiet der Triboci, ca. 50 Kilometer nördlich von Strasbourg/Argentorate, darf

\footnotetext{
${ }^{86}$ J.-J. HATT, Gallia 20/2 (1962), 504-505. Abb.47 (Photo), ders., Revue d'Alsace 104 (1966-1974), 25 26, LAZZARO 1979, 202. Nr.23, LAZZARO 1993, 166. Nr.156, FlOTTÉ/FUCHS 2000, 390. Entsprechende Lesungen bieten ferner die Datenbanken EDCS Nr.13303317 und LUPA Nr.27321 (mit Photo).

${ }^{87}$ KAJANTO 1966, 5-7.

88 Der Name wurde auch nicht von KAJANTO 1966 oder im OPEL II aufgenommen. S. aber KAKOSCHKE 2007, 319. CN 1170.
} 
man mit Vorsicht vermuten, dass der Verstorbene ein Veteran der seit vespasianischer Zeit in Strasbourg/Argentorate stationierten legio VIII Augusta war. ${ }^{89}$

Darüber hinaus scheint die Ergänzung des Gentilnomens in Zeile 1 zumindest fraglich, da der Abstand zwischen den beiden T sehr eng ist. Der Raum ist auf jeden Fall viel enger als im Namen Attiola (Zeile 2). Zudem lässt sich das in Zeile 5 gelesene DEF kaum auf den folgenden Namen beziehen. Es müsste sich vielmehr auf die zuvor genannte nurus Lea beziehen. Die Lesung des auf DEF folgenden Namens scheint unwahrscheinlich, da der Abstand zwischen L und ANO recht gering ist. An Stelle des L sollte man eher ein I lesen und somit statt des seltenen Namens Lanus ${ }^{90}$ den Namen Ianus entziffern, der im nahen Rheinzabern/Tabernae auch als Töpfername bezeugt ist. ${ }^{91}$ In der letzten Zeile steht entgegen WUILLEUMIER nicht VS SIBI, sondern VSIBI. Dabei ist zwischen VS und IBI offenbar noch schwach ein Worttrenner zu erkennen. Und am Ende von Zeile 2 schlug der Steinmetz nicht CONIVGI ein, sondern lediglich CONIVG. Schließlich beginnen und enden die durch WUILLEUMIER ergänzten Zeilen nicht auf einer Höhe. Die Überlegungen führen zu folgender Neulesung der Inschrift (Abb.10), die jedoch gleichfalls noch einige Unsicherheiten birgt. ${ }^{92}$ Die Rekonstruktion basiert auf der Annahme, dass die Platte für eine Zweitverwendung nicht auf beiden Seiten, sondern lediglich auf der linken Seite gekürzt wurde.

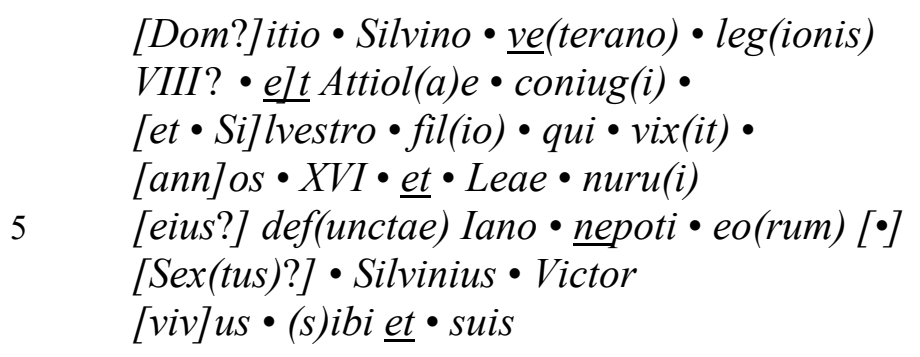

LAZZARO ordnet die Grabinschrift dem ersten oder zweiten Jahrhundert n. Chr. zu. ${ }^{93}$ Die Dativform Attiole und die Wendung qui vixit annos sprechen jedoch eher für eine Datierung in die Zeit nach $150 \mathrm{n}$. Chr. Dementsprechend kann man vermuten, dass über der Inschrift, in einer verlorengegangenen ersten Zeile, noch die Buchstaben DM für die im zweiten und dritten Jahrhundert $\mathrm{n}$. Chr. im Rheingebiet gängige Wendung Dis Manibus standen. In diesem Fall müsste man davon ausgehen, dass die Platte für eine Zweitverwendung auch im oberen Bereich gekürzt wurde.

In dem Dedikanten der Inschrift, Silvinius Victor, und seiner Familie darf man ohne Zweifel Einheimische aus den gallisch-germanischen Provinzen, möglicherweise Triboker, erblicken. Hierfür spricht vor allem das charakteristische Pseudo-

\footnotetext{
${ }^{89}$ Die Abkürzung VE statt des gängigeren VET für veteranus ist mehrfach bezeugt. Aus dem näheren Raum s. CIL XIII 7269 (Mainz-Kastel/Castellum Mattiacorum) und AE 2001, 1569 (Künzing/ Quintana). Belege für die Nennung der legio VIII ohne den Beinamen Augusta sind seltener, aber auch bezeugt: CIL XIII 2944, 2947 (beide Sens/Agedincum), 3983 (Arlon/Orolaunum).

${ }^{90} \mathrm{Zu}$ Lanus s. KAKOSCHKE 2007, 450. CN 1677, OPEL III 18 (mit den Belegen CIL XIII 286, ILTG 436), DelamarRe 2007, 115 (mit dem Beleg ILTG 436).

${ }^{91}$ Zum keltischen Ianus s. KAKOSCHKE 2007, 418. CN 1562, OPEL II 190, Delamarre 2007, 107. Zum Töpfer Ianus aus Rheinzabern/Tabernae s. HARTLEY/DICKINSON 2009, 248-251.

${ }^{92}$ So könnte z.B. in den Zeilen $5 / 6$ ebenso zu eo(rum) $[\bullet] /$ [Tib(erius)] • Silvinius bzw. zu eo/[rum] • Silvinius oder ähnlich ergänzt werden.

${ }^{93}$ LAZZARO 1993, 166. Nr.156.
} 
gentiliz des Dedikanten, das aus dem Cognomen des Vaters gebildet wurde, ${ }^{94}$ und der Name der Mutter, Attiola, der fast ausschließlich im Großraum Gallien bezeugt ist. ${ }^{95}$

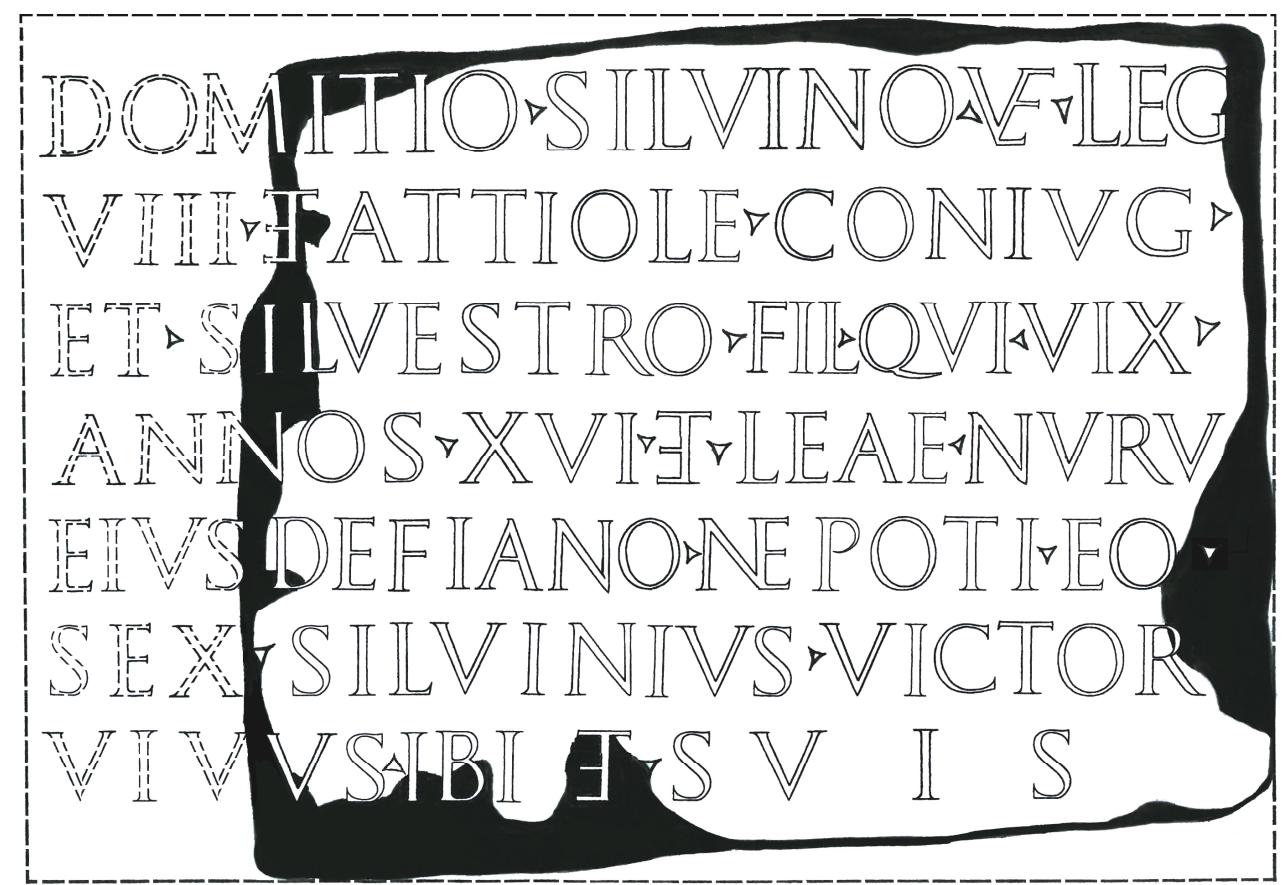

Abb.10: Leicht idealisierte Rekonstruktionszeichnung der fragmentarischen Grabinschrift ILTG 436 aus Langensoultzbach (Zeichnung A.K.)

\section{Siglen}

1. N.

2. N

3. N

4. N.

$\mathrm{AE}$

$\mathrm{AKB}$

$\mathrm{BJb}$
H. FINKE, Neue Inschriften, BRGK 17 (1927), 1-107, 198-231.

H. Nesselhauf, Neue Inschriften aus dem römischen Germanien und den angrenzenden Gebieten, BRGK 27 (1937), 51-134.

H. Nesselhauf / H. LieB, Dritter Nachtrag zu CIL. XIII. Inschriften aus den germanischen Provinzen und dem Treverergebiet, BRGK 40 (1959), 120-229.

U. SCHILlingeR-HÄFELE, Vierter Nachtrag zu CIL XIII und zweiter Nachtrag zu FR. VOLLMER, Inscriptiones Baivariae Romanae. Inschriften aus dem deutschen Anteil der germanischen Provinzen und des Treverergebietes sowie Rätiens und Noricums, BRGK 58 (1977), 447-603.

L’Année Épigraphique, Paris.

Archäologisches Korrespondenzblatt, Mainz.

Bonner Jahrbuch, Bonn.

\footnotetext{
${ }^{94} \mathrm{Zu}$ Silvinius s. KAKOSCHKE 2006, 371-372. GN 1186, OPEL IV 82-83.

95 Zum keltischen Namen Attiola s. KAKOSCHKE 2007, 135-136. CN 364, OPEL I² 90, DelamarRE 2007,32 .
} 
BRGK

CIL

CLE

CSIR

EPRO

FBW

GFA

$\mathrm{ICH}$

IKöln ${ }^{2}$

ILTG

JberAugst

JbRGZM

$\mathrm{KJb}$

$\mathrm{MZ}$

OPEL I²-IV

BAUMANN 1890

BOPPERT 1992

BRAMBACH 1867
Bericht der Römisch-Germanischen Kommission, Mainz.

Corpus Inscriptionum Latinarum, Berlin $1863 \mathrm{ff}$.

F. Buecheler, Anthologia Latina II. Carmina Latina epigraphica Fasc.1, Leipzig 1895; Fasc.3 (Supplementum von E. LOMMATZSCH), Leipzig 1926.

Corpus Signorum Imperii Romani, Mainz u.a.

Études préliminaires aux religiones orientales dans l'empire romain, Leiden.

Fundberichte aus Baden-Württemberg, Stuttgart.

Göttinger Forum für Altertumswissenschaft, Göttingen. (www.gfa.gbv.de)

TH. Mommsen, Inscriptiones Confoederationis Helveticae Latinae, Mitteilungen der Antiquarischen Gesellschaft Zürich 10, Zürich 1854.

B. GALSTERER / H. GALSTERER, Die römischen Steininschriften aus Köln. IKöln ${ }^{2}$, Kölner Forschungen 10, Mainz 2010.

P. Wuilleumier, Inscriptions latines des trois Gaules (France), Gallia Supplément 17, Paris 1963.

Jahresberichte aus Augst und Kaiseraugst, Liestal.

Jahrbuch des Römisch-Germanischen Zentralmuseums Mainz, Mainz.

Kölner Jahrbuch für Vor- und Frühgeschichte, Köln.

Mainzer Zeitschrift, Mainz.

Onomasticon Provinciarum Europae Latinarum $\mathrm{I}^{2}$-IV. Ex materia ab A. Mócsy, R. FELDMANN, E. MARTON et M. SZILÁGYI collecta, hrsg. von B. LŐRINCZ u.a., Budapest-Wien 1999-2005.

\section{Literaturverzeichnis}

K. BAumanN, Römische Denksteine und Inschriften der Vereinigten Altertums-Sammlungen in Mannheim, Wissenschaftliche Beigabe zum Programm des Gymnasiums zu Mannheim für das Schuljahr 1888/89, Mannheim.

W. BOPPERT, Militärische Grabdenkmäler aus Mainz und Umgebung, CSIR Deutschland II,5. Germania superior, Mainz.

W. BRAMBACH, Corpus Inscriptionum Rhenanarum, Elberfeld.

http://www.fera-journal.eu 
Clauss 1992

DELAMARRE 2007

DIEDENHOFEN 1974

ENGSTER 2002

ESPÉRANDIEU 1922

FLOTTÉ/FuCHS 2000

GALSTERER 1975

GARMAN 2008

GROPENGIEßER 1975

GUTENBRUNNER 1936

HAUG 1877

HETTNER 1876

HOPE 2001
M. Clauss, Cultores Mithrae. Die Anhängerschaft des Mithras-Kultes, Heidelberger althistorische Beiträge und epigraphische Studien 10, Stuttgart.

X. Delamarre, Nomina Celtica antiqua selecta inscriptionum (Noms de personnes celtiques dans l'épigraphie classique), Paris.

W. Diedenhofen, Die Antikensammlung auf Schloss Wissen (1591-1648), Geldrischer Heimatkalender 1974, 136-165.

D. EngSTER, Konkurrenz oder Nebeneinander. Mysterienkulte in der hohen römischen Kaiserzeit, Quellen und Forschungen zur antiken Welt 36, München.

É. ESPÉRANDIEU, Recueil général des bas-reliefs, statues et bustes de la Gaule romaine 8, Paris.

P. Flotté / M. Fuchs, Carte archéologique de la Gaule 67/1. Les Bas-Rhin, Paris.

B. Galsterer / H. GALSTERER, Die römischen Steininschriften aus Köln, Wissenschaftliche Kataloge des Römisch-Germanischen Museums 2, Köln.

A. G. GARMAN, The cult of the Matronae in the Roman Rhineland. An historical evalution of the archaeological evidence, Lewiston, NY.

E. GropengIEßER, Römische Steindenkmäler, Bildhefte des Städt. Reiss-Museums Mannheim. Archäologische Sammlungen 1, Mannheim.

S. GUTENBRUNNER, Die germanischen Götternamen der antiken Inschriften, Rheinische Beiträge und Hülfsbücher zur germanischen Philologie und Volkskunde 24, Halle (Saale).

Hartley/Dickinson 2009 B. R. Hartley / B. M. Dickinson, Names on Terra Sigillata. An Index of Makers' Stamps \& Signatures on Gallo-Roman Terra Sigillata (Samian Ware). Bd.4. F to KLUMI, London.

F. HAUG, Die römischen Denksteine des grossherzoglichen Antiquariums in Mannheim, Wissenschaftliche Beigabe zu den Programmen des Gymnasiums Mannheim für die Schuljahre 1875/77, Konstanz.

F. HetTneR, Katalog des Königlichen rheinischen Museums vaterländischer Altertümer bei der Universität Bonn, Bonn.

V. M. Hope, Constructing identity: The Roman funerary monuments of Aquileia, Mainz and Nîmes, BAR International Series 960, Oxford.

http://www.fera-journal.eu 
KAJANTO 1966

KAJANTO 1977

KAKOSCHKE 2006

KAKOSCHKE 2007

KAKOSCHKE 2008

KAKOSCHKE 2014

KRONEMAYER 1983

LAZZARO 1979

LAZZARO 1993

RIESE 1914

SCHALLES 2006

SCHARF 1938

SCHEID 2006
I. KAJANTO, Supernomina. A Study in Latin Epigraphy, Societas Scientiarum Fennica. Commentationes Humanarum Litterarum 40.1, Helsinki-Helsingfors.

I. Kajanto, On the Peculiarities of Women's Nomenclature, in: Actes du Colloque International sur l'Onomastique Latine organisé à Paris du 13 au 15 octobre 1975 par M. H.-G. PfLAuM et M. N. DuVAL. Éd. N. DuVAL, Paris, 147-159.

A. KakoschKe, Die Personennamen in den zwei germanischen Provinzen. Ein Katalog. Bd.1: Gentilnomina ABILIUS-VOLUSIUS, Rahden/Westf.

A. KakoschKe, Die Personennamen in den zwei germanischen Provinzen. Ein Katalog. Bd.2,1: Cognomina ABAIUS-LYSIAS, Rahden/Westf.

A. KAKoschKe, Die Personennamen in den zwei germanischen Provinzen. Ein Katalog. Bd.2,2: Cognomina MACCAUS-ZYASCELIS, Rahden/Westf.

A. KAKOSCHKE, Hapax - Steinmetzirrtum - Überlieferungsfehler - Fehllesung - Fälschung. Zu einigen auffälligen Personennamen aus den zwei germanischen Provinzen, Stuttgart.

V. KRONEMAYER, Beiträge zur Sozialgeschichte des römischen Mainz, Europäische Hochschulschriften. Reihe 3. Geschichte und ihre Hilfswissenschaften 199, Frankfurt am Main-Bern-New York.

L. LAZZARO, Nouvelles données épigraphiques pour l'approche des formes de dépendance en Belgique et dans les Germanies: Supplément au CIL XIII, Dialoges d'histoire ancienne 5. 191-231.

L. LazZARO, Esclaves et affranchis. En Belgique et Germanies Romaines d'après les sources épigraphiques, Annales Littéraires de l'Université de Besançon 430, Paris.

A. RIESE, Das rheinische Germanien in den antiken Inschriften, Leipzig-Berlin.

H.-J. Schalles, Epigraphisches vom Niederrhein, Xantener Berichte 14, 85-129.

J. SCHARF, Studien zur Bevölkerungsgeschichte der Rheinlande auf epigraphischer Grundlage, Neue Deutsche Forschungen. Abteilung Alte Geschichte 3, Berlin.

J. SCHEID, Les dévots en Germanie inférieure: divinités, lieux de culte, fidèles, in: M. DONDIN-PAYRE / M.-TH. RAEPSAET-CHARliER (Hrsg.), Sanctuaires, pratiques

http://www.fera-journal.eu 
SCHWARZ/FURGER 1988

SCHWERTHEIM 1974

SPICKERMANN 1994

STÄHELIN 1948

VERMASEREN 1960

WAGNER 1911

WALSER 1980

WIEGELS 2010

WEISGERBER 1968

EDCS

$\mathrm{EDH}$

LUPA cultuelles et territoires civiques dans l'Occident romain, Bruxelles, 297-346.

P.-A. Schwarz / A. R. Furger, Inscriptiones Selectae Coloniae Augustae Rauricorum. Ausgewählte Inschriften aus Augst und Kaiseraugst, Augster Blätter zur Römerzeit 6, Augst.

E. Schwertheim, Die Denkmäler orientalischer Gottheiten im römischen Deutschland, EPRO 40, Leiden.

W. SPICKERMANN, „Mulieres ex Voto“. Untersuchungen zur Götterverehrung von Frauen im römischen Gallien, Germanien und Rätien (1.-3. Jahrhundert n. Chr.), Bochumer historische Studien. Alte Geschichte 12, Bochum.

F. STÄHELIN, Die Schweiz in römischer Zeit. 3., neu bearbeitete und erweiterte Aufl., Basel.

M. J. Vermaseren, Corpus Inscriptionum et Monumentorum Religionis Mithriacae. Bd.2, EPRO 50, Den Haag.

E. WAGNER, Fundstätten und Funde aus vorgeschichtlicher, römischer und alamannischer Zeit im Grossherzogtum Baden. Teil 2, Tübingen.

G. WALSER, Römische Inschriften in der Schweiz für den Schulunterricht ausgewählt, photographiert und erklärt, 2. Teil: Nordwest- und Nordschweiz, Bern.

R. WIEGELS, Kleine Schriften zur Epigraphik und Militärgeschichte der germanischen Provinzen. Hrsg. von K. MATIJEVIĆ und W. SPICKERMANN. Stuttgart.

J. L. WeISGERBER, Die Namen der Ubier, Wissenschaftliche Abhandlungen der Arbeitsgemeinschaft für Forschung des Landes Nordrhein-Westfalen 34, KölnOpladen.

\section{Elektronische Datenbanken}

Epigraphik-Datenbank Clauss/Slaby (Frankfurt), Leitung: M. Clauss (Johann Wolfgang Goethe-Universität Frankfurt am Main), Stand: 26.10.2017 (www.manfredclauss.de).

Epigraphische Datenbank Heidelberg, Leitung: $\mathrm{CH}$. WITSCHEL (Ruprecht-Karls-Universität Heidelberg), Stand: 26.10.2017 (www.uni-heidelberg.de/institute/ sonst/adw/edh).

UBI ERAT LUPA, Bilddatenbank zu antiken Steindenkmälern (Universität Salzburg), Leitung: F. und O. HARL

http://www.fera-journal.eu 
Frankfurter elektronische Rundschau zur Altertumskunde 34 (2017)

(Universität Wien), Stand: 26.10.2017 (www.ubi-eratlupa.org).

\section{Kontakt zum Autor:}

Dr. Andreas Kakoschke

Nelly-Sachs-Weg 1

D-49191 Belm

E-Mail: andreaskakoschke@,hotmail.de 